\title{
Dimension free and infinite variance tail estimates on Poisson space
}

\author{
Jean-Christophe Breton Christian Houdré Nicolas Privault
}

\begin{abstract}
Concentration inequalities are obtained on Poisson space, for random functionals with finite or infinite variance. In particular, dimension free tail estimates and exponential integrability results are given for the Euclidean norm of vectors of independent functionals. In the finite variance case these results are applied to infinitely divisible random variables such as quadratic Wiener functionals, including Lévy's stochastic area and the square norm of Brownian paths. In the infinite variance case, various tail estimates such as stable ones are also presented.
\end{abstract}

Key words: Concentration, infinite divisibility, stable laws, Poisson space, OrnsteinUhlenbeck semi-group, quadratic Wiener functionals, large deviations. Mathematics Subject Classification: 60F99, 60E07, 60G57.

\section{Introduction and notation}

Let $\Omega^{X}$ denote the set of Radon measures

$$
\Omega^{X}=\left\{\omega=\sum_{i=1}^{N} \epsilon_{t_{i}}:\left(t_{i}\right)_{i=1}^{i=N} \subset X, t_{i} \neq t_{j}, \forall i \neq j, N \in \mathbb{N} \cup\{\infty\}\right\},
$$

where $X$ is a $\sigma$-compact metric space with distance $d_{X}$, and $\epsilon_{t}$ denotes the Dirac measure at $t \in X$. Let $\nu$ be a diffuse Radon measure on $X$, and let $P$ be the Poisson measure with intensity $\nu$ on $\Omega^{X}$. Let the linear, closable, finite difference operator

$$
D: L^{2}\left(\Omega^{X}, P\right) \longrightarrow L^{2}\left(\Omega^{X} \times X, P \otimes \nu\right)
$$

be defined via

$$
D_{x} F(\omega)=F(\omega \cup\{x\})-F(\omega), \quad d P \times \nu(d \omega, d x) \text {-a.e. }
$$


where as a convention we identify $\omega \in \Omega^{X}$ with its support, cf. e.g. [20], [22], 23].

In [1] 27], 8], Poisson tail estimates are obtained under the hypothesis

$$
D F \leq K, \quad P \otimes \nu \text {-a.e., } \quad \text { and } \quad\|D F\|_{L^{\infty}\left(\Omega^{X}, L^{2}(X, \nu)\right)} \leq \tilde{\alpha}<\infty
$$

for some $K \geq 0$. While (modified) logarithmic Sobolev inequalities and the Herbst method are used in [1] and 27], the methods of [8] rely on covariance representations (4], 6] $)$. Recently the results of [6] have further led in [7] to estimates for Lipschitz functions of stable random vectors. Even more recently, dimension free concentration is obtained in [9] for the Euclidean norm as well as for various classes of functions of independent infinitely divisible vectors having finite exponential moments.

In the present paper we first obtain new deviation inequalities on Poisson space via the covariance method. Then, by replacing the bounds on $D F$ and on $\|D F\|_{L^{\infty}\left(\Omega^{X}, L^{2}(X, \nu)\right)}$ by growth conditions, deviation results for Poisson functionals with infinite variance are given.

Let us briefly describe the content of the paper. In Section 2 we deal with Lévy measures with finite variance, using the covariance representation method involving the Ornstein-Uhlenbeck semi-group. This leads to general deviation results for Poisson functionals having finite exponential moments. In Section 3 we obtain dimension free deviation estimates and exponential integrability properties for random vectors of such Poisson functionals. Since an infinitely divisible random vector can be represented as a vector of Poisson stochastic integrals, these results are then applied to derive deviation inequalities for Lipschitz functions of infinitely divisible vectors. In Section 4, we study the particular case of quadratic Wiener functionals, including the square norm of Brownian path, the sample variance of Brownian motion and Lévy's stochastic area. For such i.i.d. vectors, this also gives dimension free inequalities in Euclidean norm, and large deviation estimates in $\ell^{p}$-norm, $p \in[1, \infty]$, recovering tail estimates of [2] for non-decoupled Gaussian chaos of degree 2. In Section [5] we adapt the method of [7] to prove other tail estimates under weaker hypothesis on the gradient. For example, if $\nu$ is the Lévy measure of an $\alpha$-stable vector, the bounds on $D$ can be replaced by 
the growth conditions

$$
\sup _{x \in B_{X}(0, R)}\left|D_{x} F\right| \leq C^{\prime} R \quad \text { and } \quad\|D F\|_{L^{\infty}\left(\Omega^{X}, L^{2}\left(B_{X}(0, R)\right)\right)}^{2} \leq C R^{2-\alpha}, \quad R \geq R_{0},
$$

where $B_{X}(0, R)=\left\{x \in X: d_{X}(0, x) \leq R\right\}$ is the ball of radius $R$ in $X$. Here, 0 denotes a fixed arbitrary center in $X$, whose choice has no influence on the growth conditions (1.1). This leads to an estimate of stable type for the deviation of $F$ from one of its medians.

Let us now introduce some notation which will be used throughout the paper. The multiple Poisson stochastic integral $I_{n}\left(f_{n}\right)$ is defined as

$$
I_{n}\left(f_{n}\right)(\omega)=\int_{\Delta_{n}} f_{n}\left(y_{1}, \ldots, y_{n}\right)\left(\omega\left(d y_{1}\right)-\nu\left(d y_{1}\right)\right) \cdots\left(\omega\left(d y_{n}\right)-\nu\left(d y_{n}\right)\right),
$$

for every square-integrable symmetric function $f_{n} \in L^{2}(X, \nu)^{\circ n}$, where

$$
\Delta_{n}=\left\{\left(x_{1}, \ldots, x_{n}\right) \in X^{n}: x_{i} \neq x_{j}, \forall i \neq j\right\}
$$

Recall the isometry formula

$$
E\left[I_{n}\left(f_{n}\right) I_{m}\left(g_{m}\right)\right]=n ! 1_{\{n=m\}}\left\langle f_{n}, g_{m}\right\rangle_{L^{2}(X, \nu)^{\circ n}}
$$

see [21], and recall also that every square-integrable random variable $F \in L^{2}\left(\Omega^{X}, P\right)$ admits the Wiener-Poisson decomposition

$$
F=\sum_{n=0}^{\infty} I_{n}\left(f_{n}\right) .
$$

The operator $D$ defined above is such that

$$
D_{x} I_{n}\left(f_{n}\right)(\omega)=n I_{n-1}\left(f_{n}(*, x)\right)(\omega), \quad P(d \omega) \otimes \nu(d x) \text {-a.e., } \quad n \in \mathbb{N},
$$

and in particular,

$$
D_{x} I_{1}(f)(\omega)=f(x), \quad \nu(d x) \text {-a.e. }
$$

We denote by $\operatorname{Dom}(\mathrm{D})$ the domain of $D$, i.e. the space of functionals $F \in L^{2}\left(\Omega^{X}, P\right)$ such that $D F \in L^{2}\left(\Omega^{X} \times X, P \otimes \nu\right)$. Recall also that the Ornstein-Uhlenbeck semigroup $\left(P_{t}\right)_{t \in \mathbb{R}_{+}}$is defined via

$$
P_{t} I_{n}\left(f_{n}\right)=e^{-n t} I_{n}\left(f_{n}\right), \quad f_{n} \in L^{2}(X, \nu)^{\circ n}, \quad n \in \mathbb{N} .
$$


In the sequel we also use the integral representation of the Ornstein-Uhlenbeck semigroup $\left(P_{t}\right)_{t \in \mathbb{R}_{+}}$in terms of a probability kernel $p_{t}(\omega, d \tilde{\omega}, d \hat{\omega})$, cf. e.g. [26]:

$$
P_{t} F(\omega)=\int_{\Omega^{X} \times \Omega^{X}} F(\tilde{\omega} \cup \hat{\omega}) p_{t}(\omega, d \tilde{\omega}, d \hat{\omega})
$$

When $X=\mathbb{R}^{n},|\cdot|_{p}$ denotes the $\ell^{p}$-norm on $\mathbb{R}^{n}, p \geq 1$. Assuming that

$$
\int_{\mathbb{R}^{n}} 1 \wedge|y|_{2}^{2} \nu(d y)<\infty
$$

any $n$-dimensional infinitely divisible (ID) random vector $F=\left(F_{1}, \ldots, F_{n}\right)$ without Gaussian component and with Lévy measure $\nu$ can be represented as the vector of single Poisson stochastic integrals

$$
F=\left(\int_{\left\{|y|_{2} \leq 1\right\}} y_{k}(\omega(d y)-\nu(d y))+\int_{\left\{|y|_{2}>1\right\}} y_{k} \omega(d y)+b_{k}\right)_{1 \leq k \leq n}
$$

for some $b \in \mathbb{R}^{n}$. Indeed, the characteristic function of $F$ is given by

$$
\varphi_{F}(u)=E\left[e^{i\langle F, u\rangle}\right]=\exp \left(i\langle b, u\rangle+\int_{\mathbb{R}^{n}}\left(e^{i\langle y, u\rangle}-1-i\langle y, u\rangle \mathbf{1}_{\left\{|y|_{2} \leq 1\right\}}\right) \nu(d y)\right),
$$

$u \in \mathbb{R}^{n}$.

\section{Deviation results from the Ornstein-Uhlenbeck semi-group}

As in [8], we need the following covariance identity on Poisson space, which is obtained from the Ornstein-Uhlenbeck semi-group.

Lemma 2.1 Let $F, G \in \operatorname{Dom}(\mathrm{D})$, then

$$
\operatorname{Cov}(F, G)=E\left[\int_{0}^{\infty} e^{-s} \int_{X} D_{y} F P_{s} D_{y} G \nu(d y) d s\right] .
$$

Proof. By orthogonality of multiple integrals of different orders and continuity of $P_{s}$, $s \in \mathbb{R}_{+}$, on $L^{2}\left(\Omega^{X}, P\right)$, it suffices to prove the identity for $F=I_{n}\left(f_{n}\right)$ and $G=I_{n}\left(g_{n}\right)$ :

$$
\begin{aligned}
E\left[I_{n}\left(f_{n}\right) I_{n}\left(g_{n}\right)\right] & =n !\left\langle f_{n}, g_{n}\right\rangle_{L^{2}(X, \nu)^{\circ n}}=n ! \int_{X^{n}} f_{n} g_{n} d \nu^{\otimes n} \\
& =n ! \int_{X} \int_{X^{(n-1)}} f_{n}(x, y) g_{n}(x, y) \nu^{\otimes(n-1)}(d x) \nu(d y)
\end{aligned}
$$




$$
\begin{aligned}
& =n \int_{X} E\left[I_{n-1}\left(f_{n}(\cdot, y)\right) I_{n-1}\left(g_{n}(\cdot, y)\right)\right] \nu(d y) \\
& =\frac{1}{n} E\left[\int_{X} D_{y} I_{n}\left(f_{n}\right) D_{y} I_{n}\left(g_{n}\right) \nu(d y)\right] \\
& =E\left[\int_{0}^{\infty} e^{-n s} \int_{X} D_{y} I_{n}\left(f_{n}\right) D_{y} I_{n}\left(g_{n}\right) \nu(d y) d s\right] \\
& =E\left[\int_{0}^{\infty} e^{-s} \int_{X} D_{y} I_{n}\left(f_{n}\right) P_{s} D_{y} I_{n}\left(g_{n}\right) \nu(d y) d s\right] .
\end{aligned}
$$

Using the covariance identity (2.1) and the representation (1.2) we first state a general deviation result which slightly improves the one presented in [8]. In particular it will be applied, in Section 3, to obtain deviation inequalities on product spaces for vectors of random functionals. In this proposition and the following ones, the supremum on $\Omega^{X}$ can be taken as an essential supremum with respect to $P$.

Proposition 2.2 Let $F \in \operatorname{Dom}(\mathrm{D})$ be such that $e^{s F} \in \operatorname{Dom}(\mathrm{D}), 0 \leq s \leq t_{0}$, for some $t_{0}>0$. Then

$$
P(F-E[F] \geq x) \leq \exp \left(\min _{0<t<t_{0}} \int_{0}^{t} h(s) d s-t x\right), \quad x>0,
$$

where

$$
h(s)=\sup _{\left(\omega, \omega^{\prime}\right) \in \Omega^{X} \times \Omega^{X}}\left|\int_{X}\left(e^{s D_{y} F(\omega)}-1\right) D_{y} F\left(\omega^{\prime}\right) \nu(d y)\right|, \quad s \in\left[0, t_{0}\right) .
$$

If moreover $h$ is nondecreasing and finite on $\left[0, t_{0}\right)$ then

$$
P(F-E[F] \geq x) \leq \exp \left(-\int_{0}^{x} h^{-1}(s) d s\right), \quad 0<x<h\left(t_{0}^{-}\right),
$$

where $h^{-1}$ is the left-continuous inverse of $h$ :

$$
h^{-1}(x)=\inf \{t>0: h(t) \geq x\}, \quad 0<x<h\left(t_{0}^{-}\right) .
$$

Proof. We start by deriving the following inequality for a centered random variable $F$ :

$$
E\left[F e^{s F}\right] \leq h(s) E\left[e^{s F}\right], \quad 0 \leq s \leq t_{0}
$$


This follows from (2.1). Indeed, using the integral representation (1.2) of the OrnsteinUhlenbeck semi-group $\left(P_{t}\right)_{t}$ for $P_{v} D_{y} F(\omega)$, we have

$$
\begin{aligned}
E & {\left[F e^{s F}\right]=E\left[\int_{0}^{\infty} e^{-v} \int_{X} D_{y} e^{s F} P_{v} D_{y} F \nu(d y) d v\right] } \\
& =\int_{\Omega^{X}} \int_{0}^{\infty} e^{-v} \int_{X}\left(e^{s D_{y} F(\omega)}-1\right) e^{s F(\omega)} \int_{\Omega^{X} \times \Omega^{X}} D_{y} F(\tilde{\omega} \cup \hat{\omega}) p_{v}(\omega, d \tilde{\omega}, d \hat{\omega}) \nu(d y) d v P(d \omega) \\
& \leq \int_{\Omega^{X}} \int_{0}^{\infty} e^{-v} e^{s F(\omega)} \int_{\Omega^{X} \times \Omega^{X}}\left|\int_{X}\left(e^{s D_{y} F(\omega)}-1\right) D_{y} F(\tilde{\omega} \cup \hat{\omega}) \nu(d y)\right| p_{v}(\omega, d \tilde{\omega}, d \hat{\omega}) d v P(d \omega) \\
& \leq \sup _{\left(\omega, \omega^{\prime}\right) \in \Omega^{X} \times \Omega^{X}}\left|\int_{X}\left(e^{s D_{y} F(\omega)}-1\right) D_{y} F\left(\omega^{\prime}\right) \nu(d y)\right| E\left[e^{s F} \int_{0}^{\infty} e^{-v} d v\right] \\
& =\sup _{\left(\omega, \omega^{\prime}\right) \in \Omega^{X} \times \Omega^{X}}\left|\int_{X}\left(e^{s D_{y} F(\omega)}-1\right) D_{y} F\left(\omega^{\prime}\right) \nu(d y)\right| E\left[e^{s F}\right]
\end{aligned}
$$

which yields (2.4) . In the general case, we let $L(s)=E\left[e^{s(F-E[F])}\right]$ and obtain:

$$
\frac{L^{\prime}(s)}{L(s)} \leq h(s), \quad 0 \leq s \leq t_{0}
$$

which using Chebychev's inequality gives:

$$
P(F-E[F] \geq x) \leq \exp \left(-t x+\int_{0}^{t} h(s) d s\right) .
$$

Using the relation $\frac{d}{d t}\left(\int_{0}^{t} h(s) d s-t x\right)=h(t)-x$, we can then optimize as follows:

$$
\begin{aligned}
\min _{0<t<t_{0}} \int_{0}^{t} h(s) d s-t x & =\int_{0}^{h^{-1}(x)} h(s) d s-x h^{-1}(x) \\
& =\int_{0}^{x} s d h^{-1}(s)-x h^{-1}(x) \\
& =-\int_{0}^{x} h^{-1}(s) d s,
\end{aligned}
$$

hence

$$
P(F-E[F] \geq x) \leq \exp \left(-\int_{0}^{x} h^{-1}(s) d s\right), \quad 0<x<h\left(t_{0}^{-}\right)
$$

In the sequel we derive several corollaries from Proposition 2.2 and discuss possible choices for the function $h$, in particular for vectors of random functionals. Note that since

$$
h(t) \leq \int_{X}\left\|D_{y} F\right\|_{\infty}\left\|e^{t\left|D_{y} F\right|}-1\right\|_{\infty} \nu(d y),
$$


Proposition 2.2 recovers Proposition 3.3 in [8], which is obtained via a covariance identity relying on the Clark formula. In the next proposition and following [9], we obtain a better result by applying Proposition 2.2 with more careful bounds.

Proposition 2.3 Let $F: \Omega^{X} \rightarrow \mathbb{R}$ and let $K: X \rightarrow \mathbb{R}_{+}$be a non-negative function such that

$$
D_{y} F(\omega) \leq K(y), \quad y \in X, \omega \in \Omega^{X}
$$

Then

where

$$
P(F-E[F] \geq x) \leq \exp \left(\min _{t>0} \int_{0}^{t} h(s) d s-t x\right), \quad x>0,
$$

$$
h(t)=\sup _{\omega \in \Omega^{X}} \int_{X} \frac{e^{t K(y)}-1}{K(y)}\left|D_{y} F(\omega)\right|^{2} \nu(d y), \quad t>0 .
$$

If moreover $h$ is finite on $\left[0, t_{0}\right)$ then

$$
P(F-E[F] \geq x) \leq \exp \left(-\int_{0}^{x} h^{-1}(s) d s\right), \quad 0<x<h\left(t_{0}^{-}\right) .
$$

If $K(y)=0, y \in X$, we have:

$$
P(F-E[F] \geq x) \leq \exp \left(-\frac{x^{2}}{2 \tilde{\alpha}^{2}}\right), \quad x>0,
$$

with

$$
\tilde{\alpha}^{2}=\sup _{\omega \in \Omega^{X}} \int_{X}\left|D_{y} F(\omega)\right|^{2} \nu(d y)
$$

Proof. Since when $K$ is $\mathbb{R}_{+}$-valued the condition $D_{y} F_{n}(\omega) \leq K(y), \omega \in \Omega^{X}, y \in X$, is satisfied we may apply Proposition 2.2 to $F_{n}=\max (-n, \min (F, n)), n \geq 1$, and get

$$
\begin{aligned}
h(t) & =\sup _{\left(\omega, \omega^{\prime}\right) \in \Omega^{X} \times \Omega^{X}}\left|\int_{X} \frac{e^{t D_{y} F_{n}(\omega)}-1}{D_{y} F_{n}(\omega)} D_{y} F_{n}(\omega) D_{y} F_{n}\left(\omega^{\prime}\right) \nu(d y)\right| \\
& \leq \sup _{\left(\omega, \omega^{\prime}\right) \in \Omega^{X} \times \Omega^{X}} \int_{X} \frac{e^{t K(y)}-1}{K(y)}\left|D_{y} F_{n}(\omega)\right|\left|D_{y} F_{n}\left(\omega^{\prime}\right)\right| \nu(d y) \\
& \leq \frac{1}{2} \sup _{\left(\omega, \omega^{\prime}\right) \in \Omega^{X} \times \Omega^{X}} \int_{X} \frac{e^{t K(y)}-1}{K(y)}\left(\left|D_{y} F_{n}(\omega)\right|^{2}+\left|D_{y} F_{n}\left(\omega^{\prime}\right)\right|^{2}\right) \nu(d y) \\
& \leq \sup _{\omega \in \Omega^{X}} \int_{X} \frac{e^{t K(y)}-1}{K(y)}\left|D_{y} F_{n}(\omega)\right|^{2} \nu(d y) \\
& \leq \sup _{\omega \in \Omega^{X}} \int_{X} \frac{e^{t K(y)}-1}{K(y)}\left|D_{y} F(\omega)\right|^{2} \nu(d y),
\end{aligned}
$$

which allows to conclude. 
Note that if $K: X \rightarrow \mathbb{R}$ in (2.7) is not necessarily positive and $F, e^{s F} \in \operatorname{Dom}(\mathrm{D})$, $0 \leq s \leq t_{0}$, for some $t_{0}>0$, then applying Proposition 2.2 and the above argument directly to $F$ yields:

$$
P(F-E[F] \geq x) \leq \exp \left(\min _{0<t<t_{0}} \int_{0}^{t} h(s) d s-t x\right), \quad x>0,
$$

and (2.9) also holds provided $h$ is finite on $\left[0, t_{0}\right)$.

Part of the next corollary recovers a result of [27] (see also [8]). This result is used in Corollary 2.6] below as well as in the infinite variance case in Section 5.

Corollary 2.4 Let $F \in L^{2}\left(\Omega^{X}, P\right)$ be such that $D F \leq K, P \otimes \nu$-a.e., for some $K \in \mathbb{R}$, and $\|D F\|_{L^{\infty}\left(\Omega^{X}, L^{2}(X, \nu)\right)} \leq \tilde{\alpha}$. We have for $K>0$ :

$$
P(F-E[F] \geq x) \leq e^{x / K}\left(1+\frac{x K}{\tilde{\alpha}^{2}}\right)^{-\frac{x}{K}-\frac{\tilde{\alpha}^{2}}{K^{2}}}, \quad x>0,
$$

and for $K=0$ :

$$
P(F-E[F] \geq x) \leq \exp \left(-\frac{x^{2}}{2 \tilde{\alpha}^{2}}\right), \quad x>0 .
$$

Proof. If $K \geq 0$, let us first assume that $F$ is a bounded random variable. The function $h$ in (2.8) is such that

$$
h(t) \leq \frac{e^{t K}-1}{K}\|D F\|_{L^{\infty}\left(\Omega^{X}, L^{2}(X, \nu)\right)}^{2} \leq \tilde{\alpha}^{2} \frac{e^{t K}-1}{K}, \quad t>0 .
$$

Applying (2.5) with $\tilde{\alpha}^{2}\left(e^{t K}-1\right) / K$ gives

$$
P(F-E[F] \geq x) \leq \exp \left(-t x+\frac{\tilde{\alpha}^{2}}{K^{2}}\left(e^{t K}-t K-1\right)\right) .
$$

Optimizing in $t$ with $t=K^{-1} \log \left(1+K x / \tilde{\alpha}^{2}\right)$ (or using directly (2.3) with the inverse $\left.K^{-1} \log \left(1+K t / \tilde{\alpha}^{2}\right)\right)$ we have

$$
P(F-E[F] \geq x) \leq \exp \left(\frac{x}{K}-\left(\frac{x}{K}+\frac{\tilde{\alpha}^{2}}{K^{2}}\right) \log \left(1+\frac{x K}{\tilde{\alpha}^{2}}\right)\right),
$$

which yields (2.12), (2.11) and (2.10), depending on the value of $K$. For unbounded $F$, apply the above to $F_{n}=\max (-n, \min (F, n))$ with $\left|D F_{n}\right| \leq|D F|, n \geq 1$. Then (2.10) follows since, as $n$ goes to infinity, $F_{n}$ converges to $F$ in $L^{2}\left(\Omega^{X}\right), D F_{n}$ converges to $D F$ in $L^{2}\left(\Omega^{X}, L^{2}(X, \nu)\right)$, and $D F_{n} \leq K, n \geq 1$. The same argument applies if $K=0$. 
In case $K<0$ and $e^{t F} \in \operatorname{Dom}(\mathrm{D})$ for all $t>0$, Proposition 2.3 yields in a similar way:

$$
P(F-E[F] \geq x) \leq e^{x / K}\left(1+\frac{x K}{\tilde{\alpha}^{2}}\right)^{-\frac{x}{K}-\frac{\tilde{\alpha}^{2}}{K^{2}}}, \quad 0<x<-\frac{\tilde{\alpha}^{2}}{K} .
$$

If $F$ is an infinitely divisible random variable in $\mathbb{R}^{n}$, without Gaussian component and with Lévy measure $\nu$, the representation (1.3) shows that for any $\operatorname{Lipschitz}(c)$ function $f: \mathbb{R}^{n} \rightarrow \mathbb{R}$,

$$
\begin{aligned}
\left|D_{x} f(F)(\omega)\right| & =|f(F(\omega \cup\{x\}))-f(F(\omega))| \\
& \leq c\|F(\omega \cup\{x\})-F(\omega)\| \\
& =c\|x\|,
\end{aligned}
$$

where $\|\cdot\|$ is any norm in $\mathbb{R}^{n}$. Hence when $X=\mathbb{R}^{n}$ and $\nu$ has bounded support, Corollary 2.4 also recovers Corollary 1 of [6] with

$$
K=\inf \{r>0: \nu(\{x \in X:\|x\|>r\})=0\}
$$

and $\tilde{\alpha}^{2}=\int_{\mathbb{R}^{n}}\|y\|^{2} \nu(d y)$, i.e.

$$
P(f(F)-E[f(F)] \geq x) \leq e^{\frac{x}{c K}}\left(1+\frac{x K}{c \tilde{\alpha}^{2}}\right)^{-\frac{x}{c K}-\frac{\tilde{\alpha}^{2}}{K^{2}}}, \quad x>0 .
$$

On a product $X=\{1, \ldots, n\} \times Y$, where $Y$ is a $|\cdot|_{Y}$-normed linear space, we have the identification

$$
\Omega^{X} \simeq \Omega^{Y} \times \cdots \times \Omega^{Y}, \quad \omega=\left(\omega_{1}, \ldots, \omega_{n}\right) \in \Omega^{X}
$$

and

$$
D_{(i, y)} F(\omega)=\sum_{j=1}^{n} 1_{\{i=j\}}\left(F\left(\omega_{1}, \ldots, \omega_{i-1}, \omega_{i} \cup\{y\}, \omega_{i+1}, \ldots, \omega_{n}\right)-F\left(\omega_{1}, \ldots, \omega_{n}\right)\right),
$$

$i=1, \ldots, n, y \in Y$. Proposition 2.2 can be directly applied with $d \nu(i, y)=d \nu_{i}(y)$, $i=1, \ldots, n, y \in Y$, and

$$
h(t)=\left\|\sum_{i=1}^{n} \int_{Y}\right\| D_{(i, y)} F\left\|_{\infty}\left(e^{t\left|D_{(i, y)} F\right|}-1\right) \nu_{i}(d y)\right\|_{\infty},
$$


or if $\nu=\nu_{1}=\cdots=\nu_{n}$, with

$$
h(t)=\tilde{\beta} \int_{Y}|y|_{Y}\left(e^{t \beta|y|_{Y}}-1\right) \nu(d y),
$$

where

$$
\tilde{\beta}=\sup _{\omega, y \neq 0} \sum_{i=1}^{n} \frac{\left|D_{(i, y)} F(\omega)\right|}{|y|_{Y}}, \quad \text { and } \quad \beta=\sup _{i, \omega, y \neq 0} \frac{\left|D_{(i, y)} F(\omega)\right|}{|y|_{Y}} .
$$

In fact, a stronger result can be obtained as a corollary of Proposition 2.3,

Corollary 2.5 Let $X=\{1, \ldots, n\} \times Y$, where $Y$ is $a|\cdot|_{Y \text {-normed linear space and }}$ $d \nu(i, y)=d \nu_{i}(y), i=1, \ldots, n, y \in Y$. Let $F: \Omega^{X} \rightarrow \mathbb{R}$ and let $\beta_{i} \geq 0, i=1, \ldots, n$, be such that

$$
D_{(i, y)} F(\omega) \leq \beta_{i}|y|_{Y}, \quad i=1, \ldots, n, \quad y \in Y, \omega \in \Omega^{X} .
$$

Then

$$
P(F-E[F] \geq x) \leq \exp \left(\min _{t>0} \int_{0}^{t} h(s) d s-t x\right), \quad x>0,
$$

where

$$
h(t)=\sup _{\omega \in \Omega^{X}} \sum_{i=1}^{n} \int_{Y} \frac{e^{t \beta_{i}|y|_{Y}}-1}{\beta_{i}|y|_{Y}}\left(D_{(i, y)} F(\omega)\right)^{2} \nu_{i}(d y), \quad t>0 .
$$

If moreover $h$ is finite on $\left[0, t_{0}\right)$ then

$$
P(F-E[F] \geq x) \leq \exp \left(-\int_{0}^{x} h^{-1}(s) d s\right), \quad 0<x<h\left(t_{0}^{-}\right) .
$$

If $\beta_{i}=0, i=1, \ldots, n$, i.e. for decreasing functionals, we have:

$$
P(F-E[F] \geq x) \leq \exp \left(-\frac{x^{2}}{2 \tilde{\alpha}^{2}}\right), \quad x>0,
$$

with

$$
\tilde{\alpha}^{2}=\sup _{\omega \in \Omega^{X}} \sum_{i=1}^{n} \int_{Y}\left(D_{(i, y)} F(\omega)\right)^{2} \nu_{i}(d y) .
$$

Proof. Apply Proposition 2.3 with $K(i, y)=\left.\left.\beta_{i}\right|_{y}\right|_{Y}, 1 \leq i \leq n, y \in Y$.

As a consequence of (2.15), and if $\nu:=\nu_{1}=\cdots=\nu_{n}$, one can take:

$$
h(t)=\frac{\tilde{\alpha}^{2}}{\beta} \int_{Y}|y|_{Y}\left(e^{t \beta|y|_{Y}}-1\right) \nu(d y), \quad t \in\left[0, t_{0}\right],
$$


with

$$
\tilde{\alpha}^{2}=\sup _{\omega \in \Omega^{X}, y \neq 0} \sum_{i=1}^{n} \frac{\left|D_{(i, y)} F(\omega)\right|^{2}}{|y|_{Y}^{2}}, \quad \text { and } \quad \beta=\sup _{i, \omega, y \neq 0} \frac{\left|D_{(i, y)} F(\omega)\right|}{|y|_{Y}} \text {. }
$$

Taking

$$
h(t)=\sum_{i=1}^{n} \beta_{i} \int_{Y}|y|_{Y}\left(e^{t \beta_{i}|y|_{Y}}-1\right) \nu_{i}(d y), \quad t \in\left[0, t_{0}\right],
$$

allows to recover the bound implied by (2.14) in this case.

For example, if $n=1$ and

$$
F_{1}(\omega)=\int_{Y} u_{1}(y)(\omega(d y)-\nu(d y)), \ldots, F_{m}(\omega)=\int_{Y} u_{m}(y)(\omega(d y)-\nu(d y))
$$

are $m$ (not necessarily independent) single Poisson stochastic integrals and $F=$ $g\left(F_{1}, \ldots, F_{m}\right)$, we have

$$
\beta \leq \sup _{x_{1}, \ldots, x_{m}, y \neq 0} \frac{\left|g\left(x_{1}+u_{1}(y), \ldots, x_{m}+u_{m}(y)\right)-g\left(x_{1}, \ldots, x_{m}\right)\right|}{|y|_{Y}} .
$$

The following statement is obtained from Corollary 2.4 on a product space, in the same way as Corollary 2.5 is obtained from Proposition 2.3 ,

Corollary 2.6 Let $X=\{1, \ldots, n\} \times Y$ with $d \nu(i, y)=d \nu_{i}(y), i=1, \ldots, n, y \in Y$. Let $F$ be such that $D_{(i, y)} F(\omega) \leq K, P \otimes \nu_{i}$-a.e., $i=1, \ldots, n$, for some $K \in \mathbb{R}$ and

$$
\left\|\sum_{i=1}^{n}\right\| D_{(i, \cdot)} F\left\|_{L^{2}\left(Y ; \nu_{i}\right)}^{2}\right\|_{L^{\infty}\left(\Omega^{X}, P\right)} \leq \tilde{\alpha}^{2} .
$$

We have for $K>0$ :

$$
P(F-E[F] \geq x) \leq e^{x / K}\left(1+\frac{x K}{\tilde{\alpha}^{2}}\right)^{-\frac{x}{K}-\frac{\tilde{\alpha}^{2}}{K^{2}}}, \quad x>0,
$$

and for $K=0$ :

$$
P(F-E[F] \geq x) \leq \exp \left(-\frac{x^{2}}{2 \tilde{\alpha}^{2}}\right), \quad x>0 .
$$

Moreover if $K<0$ and $e^{t F} \in \operatorname{Dom}(\mathrm{D})$ for all $t>0$, then

$$
P(F-E[F] \geq x) \leq e^{x / K}\left(1+\frac{x K}{\tilde{\alpha}^{2}}\right)^{-\frac{x}{K}-\frac{\tilde{\alpha}^{2}}{K^{2}}}, \quad 0<x<-\frac{\tilde{\alpha}^{2}}{K} .
$$




\section{Application to random vectors}

We start by applying Corollary 2.5 and Corollary 2.6 to random vectors $\left(F_{1}, \ldots, F_{n}\right)$ on the product space $\Omega^{X} \simeq \Omega^{Y} \times \cdots \times \Omega^{Y}$ where $X=\{1, \ldots, n\} \times Y$ and $Y$ is a $|\cdot|_{Y}$-normed linear space. Corollary 2.5 yields

$$
P\left(g\left(F_{1}, \ldots, F_{n}\right)-E\left[g\left(F_{1}, \ldots, F_{n}\right)\right] \geq x\right) \leq \exp \left(-\int_{0}^{x} h^{-1}(s) d s\right),
$$

$0<x<h\left(t_{0}^{-}\right)$, where $g: \mathbb{R}^{n} \rightarrow \mathbb{R}$, provided the function

$$
h(t)=\left\|\sum_{i=1}^{n} \int_{Y}\left(D_{(i, y)} g\left(F_{1}(\omega), \ldots, F_{n}(\omega)\right)\right)^{2} \frac{e^{t \beta_{i}|y|_{Y}}-1}{\beta_{i}|y|_{Y}} \nu_{i}(d y)\right\|_{L^{\infty}\left(\Omega^{X}, P\right)}, \quad t \in\left[0, t_{0}\right],
$$

is finite on $\left[0, t_{0}\right)$, with $\beta_{i}$ as in Corollary 2.5. Several particular cases are now presented.

\section{Random vectors with independent components}

If $F_{1}, \ldots, F_{n}$ are $n$ independent random variables defined on $\Omega^{X}=\Omega^{Y} \times \cdots \times \Omega^{Y}$ with $F_{i}=F_{i}\left(\omega_{i}\right), i=1, \ldots, n$, and $g: \mathbb{R}^{n} \rightarrow \mathbb{R}$, an $\ell^{1}-\operatorname{Lipschitz}(c)$ function, we have

$$
\begin{aligned}
& \left|D_{(i, y)} g\left(F_{1}, \ldots, F_{n}\right)(\omega)\right| \\
& \quad=\left|g\left(F_{1}\left(\omega_{1}\right), \ldots, F_{i}\left(\omega_{i} \cup\{y\}\right), \ldots, F_{n}\left(\omega_{n}\right)\right)-g\left(F_{1}\left(\omega_{1}\right), \ldots, F_{n}\left(\omega_{n}\right)\right)\right| \\
& \quad \leq c\left|F_{i}\left(\omega_{i} \cup\{y\}\right)-F_{i}\left(\omega_{i}\right)\right| \\
& \quad \leq c\left|D_{y} F_{i}(\omega)\right| .
\end{aligned}
$$

Now we can take in Corollary 2.5 ,

$$
h(t) \leq c^{2} \sup _{\omega \in \Omega^{X}} \sum_{i=1}^{n} \int_{Y} \frac{e^{c t \beta_{i}|y|_{Y}}-1}{c \beta_{i}|y|_{Y}}\left(D_{y} F_{i}(\omega)\right)^{2} \nu_{i}(d y), \quad t \in\left[0, t_{0}\right]
$$

with

$$
\beta_{i}=\sup _{y \in Y, \omega_{i} \in \Omega^{Y}} \frac{\left|D_{y} F_{i}\left(\omega_{i}\right)\right|}{|y|_{Y}} .
$$

Moreover when $\nu=\nu_{1}=\cdots=\nu_{n}$, we can take in (2.16):

$$
\tilde{\alpha}^{2}=c^{2} \sup _{\omega, y \neq 0} \sum_{i=1}^{n} \frac{\left|D_{y} F_{i}(\omega)\right|^{2}}{|y|_{Y}^{2}}, \quad \text { and } \quad \beta=c \sup _{i, \omega, y \neq 0} \frac{\left|D_{y} F_{i}(\omega)\right|}{|y|_{Y}} .
$$




\section{Independent vectors of Poisson stochastic integrals}

Assume that $Y$ is a normed linear space and that $\int_{Y} 1 \wedge|y|_{Y}^{2} \nu_{i}(d y)<\infty, i=1, \ldots, n$. If $G=g\left(F_{1}, \ldots, F_{n}\right)$ where $g: \mathbb{R}^{n} \rightarrow \mathbb{R}$ and $F_{1}, \ldots, F_{n}$ are independent Poisson stochastic integrals of the form (1.3):

$$
F_{i}\left(\omega_{i}\right)=\int_{\left\{|y|_{Y} \leq 1\right\}} y\left(\omega_{i}(d y)-\nu_{i}(d y)\right)+\int_{\left\{|y|_{Y}>1\right\}} y \omega_{i}(d y)+b_{i}, \quad 1 \leq i \leq n
$$

we have

$$
D_{(i, y)} g\left(F_{1}, \ldots, F_{n}\right)=g\left(F_{1}, \ldots, F_{i}+y, \ldots, F_{n}\right)-g\left(F_{1}, \ldots, F_{n}\right)
$$

From Corollary 2.5 we have, denoting by $\left(e_{1}, \ldots, e_{n}\right)$ the canonical basis on $\mathbb{R}^{n}$ :

$$
\begin{aligned}
h(t)= & \sup _{\omega \in \Omega^{X}} \sum_{i=1}^{n} \int_{Y} \frac{e^{t \beta_{i}|y|_{Y}}-1}{\beta_{i}|y|_{Y}} \\
& \left(g\left(F_{1}\left(\omega_{1}\right), \ldots, F_{i}\left(\omega_{i}\right)+y, \ldots, F_{n}\left(\omega_{i}\right)\right)-g\left(F_{1}\left(\omega_{1}\right), \ldots, F_{i}\left(\omega_{i}\right), \ldots, F_{n}\left(\omega_{n}\right)\right)^{2} \nu_{i}(d y)\right. \\
\leq & \sup _{x \in \mathbb{R}^{n}} \sum_{i=1}^{n} \int_{Y} \frac{e^{t \beta_{i}|y|_{Y}}-1}{\beta_{i}|y|_{Y}}\left(g\left(x+y e_{i}\right)-g(x)\right)^{2} \nu_{i}(d y)
\end{aligned}
$$

which recovers Theorem 1 of [9] and (3) therein as a particular case. We may also take

$$
\tilde{\alpha}^{2}=\sup _{x, y \neq 0} \sum_{i=1}^{n} \frac{\left|g\left(x+y e_{i}\right)-g(x)\right|^{2}}{|y|_{Y}^{2}}, \quad \text { and } \quad \beta=\sup _{i, x, y \neq 0} \frac{\left|g\left(x+y e_{i}\right)-g(x)\right|}{|y|_{Y}}
$$

in (2.16). If $g: \mathbb{R}^{n} \rightarrow \mathbb{R}$ is $\ell^{1}-\operatorname{Lipschitz}(c)$, then $\beta=c$, and so (2.17) gives:

$$
h(t)=c \sum_{i=1}^{n} \int_{Y}|y|_{Y}\left(e^{t c|y|_{Y}}-1\right) \nu_{i}(d y), \quad t \in\left[0, t_{0}\right]
$$

For $g(x)=\sup \left(x_{1}, \ldots, x_{n}\right)$, with $Y=\mathbb{R}, \beta=1$ and

$$
\begin{aligned}
& D_{(i, y)} g\left(F_{1}, \ldots, F_{n}\right)= \begin{cases}0, & y \leq \sup \left(F_{1}, \ldots, F_{n}\right)-F_{i}, \\
F_{i}+y-\sup \left(F_{1}, \ldots, F_{n}\right), & y>\sup \left(F_{1}, \ldots, F_{n}\right)-F_{i},\end{cases} \\
& i=1, \ldots, n, y \in \mathbb{R} . \text { Hence (2.15) leads to } \\
& h(t)=\sup _{\omega \in \Omega^{x}} \sum_{i=1}^{n} \int_{\mathbb{R}} \frac{e^{t|y|}-1}{|y|}
\end{aligned}
$$




$$
\begin{aligned}
& \left(g\left(F_{1}\left(\omega_{1}\right), \ldots, F_{i}\left(\omega_{i}\right)+y, \ldots, F_{n}\left(\omega_{i}\right)\right)-g\left(F_{1}\left(\omega_{1}\right), \ldots, F_{i}\left(\omega_{i}\right), \ldots, F_{n}\left(\omega_{n}\right)\right)^{2} \nu_{i}(d y)\right. \\
= & \sup _{\omega \in \Omega^{X}} \sum_{i=1}^{n} \int_{\sup \left(F_{1}, \ldots, F_{n}\right)-F_{i}}^{\infty} \frac{e^{t|y|}-1}{|y|}\left(F_{i}+y-\sup \left(F_{1}, \ldots, F_{n}\right)\right)^{2} \nu_{i}(d y) \\
\leq & \sum_{i=1}^{n} \int_{0}^{\infty} y\left(e^{t y}-1\right) \nu_{i}(d y) .
\end{aligned}
$$

Note that in (3.3) the constants $\tilde{\alpha}^{2}$ and $\beta$ can be computed in terms of the Lipschitz constant of $g$ with respect to the $\ell^{1}$-norm. This however does not lead to dimension free estimates. Next, we show, using (3.2), that dimension free estimates can be obtained when $g$ is the Euclidean norm on $\mathbb{R}^{n}$. The other results of [9] can similarly be generalized to the present framework.

\section{Dimension free inequalities for random vectors}

Dimension free inequalities for $\ell^{2}$-Lipschitz functions of independent infinitely divisible random vectors with finite exponential moments have been obtained in Corollary 4 of [9]. In the next proposition we extend this result to Poisson random functionals.

Proposition 3.1 Let $f: \mathbb{R}^{n} \rightarrow \mathbb{R}$ be $\ell^{2}$-Lipschitz $(c)$, and let $F=\left(F_{1}, \ldots, F_{n}\right)$ be a vector of independent random functionals. Let

$$
\beta_{i}=\sup _{y \in Y, \omega \in \Omega^{X}} \frac{\left|D_{y} F_{i}(\omega)\right|}{|y|_{Y}}, \quad i=1, \ldots, n,
$$

and assume that

$$
\begin{aligned}
h(t)= & 8 \max _{i=1, \ldots, n} \beta_{i} \int_{Y}|y|_{Y}\left(e^{t \beta_{i}|y|_{Y}}-1\right) \nu_{i}(d y) \\
& +\frac{2 n}{\left(E\left[|F-E[F]|_{2}\right]\right)^{2}} \max _{i=1, \ldots, n} \beta_{i}^{3} \int_{Y}|y|_{Y}^{3}\left(e^{t \beta_{i}|y|_{Y}}-1\right) \nu_{i}(d y)
\end{aligned}
$$

is finite in $t \in\left[0, t_{0}\right)$. Then

$P\left(f\left(F_{1}, \ldots, F_{n}\right) \geq E\left[f\left(F_{1}, \ldots, F_{n}\right)\right]+c \sqrt{2 \sum_{i=1}^{n} \operatorname{Var} F_{i}}+c x\right) \leq \exp \left(-\int_{0}^{x} h^{-1}(s) d s\right)$

$0<x<h\left(t_{0}^{-}\right)$. 
Proof. Define $\phi: \mathbb{R}^{n} \rightarrow \mathbb{R}$ by

$$
\phi(x)=\sqrt{E\left[|x-G|_{2}^{2}\right]},
$$

where $|x|_{2}$ is the Euclidean norm of $x \in \mathbb{R}^{n}$ and $G$ is an independent copy of $F$. As in the proof of Corollary 4 in 9, we have

$$
\left|\phi\left(x+u e_{i}\right)-\phi(x)\right|^{2} \leq \frac{8 u^{2} E\left[\left(x_{i}-G_{i}\right)^{2}\right]}{E\left[|x-G|^{2}\right]}+\frac{2 u^{4}}{\sum_{k=1}^{n} \operatorname{Var} G_{k}}, \quad x \in \mathbb{R}^{n}, \quad u \in \mathbb{R} .
$$

Hence for $\phi(F)$, Corollary 2.5 applies with

$$
\begin{aligned}
h_{\phi}(t)= & \sup _{\omega \in \Omega^{X}} \sum_{i=1}^{n} \int_{Y} \frac{e^{t \beta_{i}|y|_{Y}}-1}{\beta_{i}|y|_{Y}}\left(D_{(i, y)} \phi(F(\omega))\right)^{2} \nu_{i}(d y) \\
\leq & \sup _{\omega \in \Omega^{X}} \sum_{i=1}^{n} \int_{Y} \frac{e^{t \beta_{i}|y|_{Y}}-1}{\beta_{i}|y|_{Y}} \\
& \left(\phi\left(F_{1}, \ldots, F_{i}+D_{y} F_{i}, \ldots, F_{n}\right)(\omega)-\phi\left(F_{1}, \ldots, F_{n}\right)(\omega)\right)^{2} \nu_{i}(d y) \\
\leq & \sup _{\omega \in \Omega^{X}} \sum_{i=1}^{n} \int_{Y} \frac{e^{t \beta_{i}|y|_{Y}}-1}{\beta_{i}|y|_{Y}}\left(8\left|D_{y} F_{i}(\omega)\right|^{2} \frac{E_{G}\left[\left(F_{i}(\omega)-G_{i}\right)^{2}\right]}{E_{G}\left[|F(\omega)-G|^{2}\right]}+\frac{2\left|D_{y} F_{i}(\omega)\right|^{4}}{\sum_{k=1}^{n} \operatorname{Var} G_{k}}\right) \nu_{i}(d y) \\
\leq & 8 \sup _{\omega \in \Omega^{X}} \max _{i=1, \ldots, n} \int_{Y} \frac{e^{t \beta_{i}|y|_{Y}}-1}{\beta_{i}|y|_{Y}}\left|D_{y} F_{i}(\omega)\right|^{2} \nu_{i}(d y) \\
& +\frac{2}{\sum_{k=1}^{n} \operatorname{Var} G_{k}} \sup _{\omega \in \Omega^{X}} \sum_{i=1}^{n} \int_{Y} \frac{e^{\left.t \beta_{i}|y|\right|_{Y}}-1}{\beta_{i}|y|_{Y}}\left|D_{y} F_{i}(\omega)\right|^{4} \nu_{i}(d y) \\
\leq & 8 \max _{i=1, \ldots, n} \beta_{i} \int_{Y}|y|_{Y}\left(e^{t \beta_{i}|y|_{Y}}-1\right) \nu_{i}(d y) \\
& +\frac{2}{\sum_{k=1}^{n} \operatorname{Var} G_{k}} \sum_{i=1}^{n} \beta_{i}^{3} \int_{Y}|y|_{Y}^{3}\left(e^{t \beta_{i}|y|_{Y}}-1\right) \nu_{i}(d y), \quad t \in\left[0, t_{0}\right] .
\end{aligned}
$$

Finally, Corollary 2.5 with the bounds

$$
E[\phi(F)] \leq \sqrt{2 \sum_{k=1}^{n} \operatorname{Var} G_{k}},
$$

and $|f(x)-E[f(F)]| \leq c \phi(x)$, yields (3.8)).

The function $h$ in (3.7) is bounded independently of the dimension $n$ if $\left(F_{1}, \ldots, F_{n}\right)$ are i.i.d., since

$$
n \min _{1 \leq k \leq n}\left(E\left[\left|F_{k}\right|\right]\right)^{2} \leq\left(E\left[|F|_{2}\right]\right)^{2} \leq n \max _{1 \leq k \leq n} E\left[\left|F_{k}\right|^{2}\right] .
$$


For the Euclidean norm of independent infinitely divisible random vectors with finite exponential moments, better results have been obtained in Corollary 3 of [9]. In the next proposition we extend this result to Poisson random functionals.

Proposition 3.2 Let $F=\left(F_{1}, \ldots, F_{n}\right)$ be a vector of independent random functionals, and let

$$
\beta_{i}=\sup _{y \in Y, \omega \in \Omega^{X}} \frac{\left|D_{y} F_{i}(\omega)\right|}{|y|_{Y}}, \quad i=1, \ldots, n,
$$

and assume that

$$
\begin{aligned}
h(t)= & 8 \max _{i=1, \ldots, n} \beta_{i} \int_{Y}|y|_{Y}\left(e^{t \beta_{i}|y|_{Y}}-1\right) \nu_{i}(d y) \\
& +\frac{2 n}{\left(E\left[|F|_{2}\right]\right)^{2}} \max _{i=1, \ldots, n} \beta_{i}^{3} \int_{Y}|y|_{Y}^{3}\left(e^{t \beta_{i}|y|_{Y}}-1\right) \nu_{i}(d y)
\end{aligned}
$$

is finite in $t \in\left[0, t_{0}\right)$. Then

$$
P\left(\left|\left(F_{1}, \ldots, F_{n}\right)\right|_{2} \geq 2 E\left[\left|\left(F_{1}, \ldots, F_{n}\right)\right|_{2}\right]+x\right) \leq \exp \left(-\int_{0}^{x} h^{-1}(s) d s\right),
$$

$0<x<h\left(t_{0}^{-}\right)$

Proof. Let $f(x)=\left(|x|_{2}-E\left[|F|_{2}\right]\right)^{+}, x \in \mathbb{R}^{n}$. From 9] we have the inequality

$$
\left|f\left(x+u e_{i}\right)-f(x)\right|^{2} \leq 8|u|^{2} \frac{\left|x_{i}\right|^{2}}{|x|_{2}^{2}}+\frac{2|u|^{4}}{\left(E\left[|F|_{2}\right]\right)^{2}}, \quad x \in \mathbb{R}^{n}, \quad u \in \mathbb{R} .
$$

Hence for $f(F)$, repeating the bounds in the proof of Proposition 3.1] we get

$$
\begin{aligned}
h(t) \leq & 8 \max _{i=1, \ldots, n} \beta_{i} \int_{Y}|y|_{Y}\left(e^{t \beta_{i}|y|_{Y}}-1\right) \nu_{i}(d y) \\
& +\frac{2}{\left(E\left[|F|_{2}\right]\right)^{2}} \sum_{i=1}^{n} \beta_{i}^{3} \int_{Y}|y|_{Y}^{3}\left(e^{t \beta_{i}|y|_{Y}}-1\right) \nu_{i}(d y), \quad t \in\left[0, t_{0}\right] .
\end{aligned}
$$

Finally, using $|x|_{2}-E\left[|F|_{2}\right] \leq\left(|x|_{2}-E\left[|F|_{2}\right]\right)^{+}$and $E\left[\left(|F|_{2}-E\left[|F|_{2}\right]\right)^{+}\right] \leq E\left[|F|_{2}\right]$ gives (3.9) for $g(F)=|F|_{2}$.

Similarly to Proposition 3.1, the deviation result of (3.9) is dimension free if $\left(F_{1}, \ldots, F_{n}\right)$ are i.i.d.

Next, we obtain a dimension free deviation for the Euclidean norm of a vector of $n$ i.i.d. random functionals with bounded support. The non-identically distributed case is done similarly, while for single integrals it is in $[9]$. 
Corollary 3.3 Let $\nu=\nu_{1}=\cdots=\nu_{n}$ have bounded support in $B_{Y}(0, R)$, let $\beta=$ $\beta_{1}=\cdots=\beta_{n}$, and let $F=\left(F_{1}, \ldots, F_{n}\right)$ be an i.i.d. vector. Then, for all $x>0$,

$$
P\left(|F|_{2} \geq x+2 E\left[|F|_{2}\right]\right) \leq \exp \left(\frac{x}{\beta R}-\left(\frac{x}{\beta R}+\frac{\tilde{\alpha}_{R}^{2}}{\beta^{2} R^{2}}\right) \log \left(1+\frac{x \beta R}{\tilde{\alpha}_{R}^{2}}\right)\right),
$$

where

$$
\tilde{\alpha}_{R}^{2}=\left(8 \beta^{2}+\frac{2 \beta^{5} R^{2}}{\left(E\left[\left|F_{1}\right|\right]\right)^{2}}\right) \int_{Y}|y|_{Y}^{2} \nu(d y) .
$$

Proof. Apply Proposition 3.2 with

$$
h(t) \leq\left(\frac{8 \beta}{R}+\frac{2 n \beta^{4} R}{\left(E\left[|F|_{2}\right]\right)^{2}}\right)\left(e^{t \beta R}-1\right) \int_{Y}|y|_{Y}^{2} \nu(d y) \leq \tilde{\alpha}_{R}^{2} \frac{e^{t \beta R}-1}{\beta R},
$$

and compute explicitly the right hand side of (3.9).

The following result yields an exponential integrability property, independent of $n$ for the $\ell^{2}$-norm of infinitely divisible random vector whose Lévy measures have bounded supports. The non identically distributed case is similar. For independent infinitely divisible random variables an analog result is obtained in [9].

Corollary 3.4 Let $F=\left(F_{1}, \ldots, F_{n}\right)$ be as in Corollary 3.3 then for all $\lambda$, with $0<$ $\lambda<\beta^{2} R^{2} /\left(e \tilde{\alpha}_{R}^{2}\right)$, we have:

$$
E\left[\exp \left(\frac{|F|_{2}}{\beta R} \log _{+} \frac{\lambda|F|_{2}}{\beta R}\right)\right]<\infty,
$$

with $\log _{+} x=\max (\log x, 0), x>0$.

Proof. Let $\lambda<\beta^{2} R^{2} /\left(e \tilde{\alpha}_{R}^{2}\right)$. We have, using (3.10):

$$
\begin{aligned}
& E\left[\exp \left(\frac{|F|_{2}}{\beta R} \log _{+} \frac{\lambda|F|_{2}}{\beta R}\right)\right]=\int_{0}^{\infty} P\left(\exp \left(\frac{|F|_{2}}{\beta R} \log _{+} \frac{\lambda|F|_{2}}{\beta R}\right) \geq t\right) d t \\
& \quad=\int_{-\infty}^{\infty} e^{y} P\left(\frac{|F|_{2}}{\beta R} \log _{+} \frac{\lambda|F|_{2}}{\beta R} \geq y\right) d y \\
& \leq 1+\int_{0}^{\infty} e^{y} P\left(\frac{|F|_{2}}{\beta R} \log \frac{\lambda|F|_{2}}{\beta R} \geq y\right) d y \\
& \leq 1+\int_{\beta R / \lambda}^{\infty} P\left(\frac{|F|_{2}}{\beta R} \log \frac{\lambda|F|_{2}}{\beta R} \geq \frac{x}{\beta R} \log \frac{\lambda x}{\beta R}\right) \frac{1+\log \frac{\lambda x}{\beta R}}{\beta R} e^{\frac{x}{\beta R} \log \frac{\lambda x}{\beta R}} d x \\
& \leq 1+\frac{2}{\beta R} \int_{\beta R / \lambda}^{\infty} P\left(|F|_{2} \geq x\right) e^{\frac{x}{\beta R} \log \frac{\lambda x}{\beta R}} \log \frac{\lambda x}{\beta R} d x
\end{aligned}
$$




$$
\begin{aligned}
\leq & 1+\frac{2}{\beta R} \int_{\beta R / \lambda}^{\infty} e^{\frac{x-2 E\left[|F|_{2}\right]}{\beta R}} e^{\frac{x}{\beta R} \log \frac{\lambda x}{\beta R}} \log \frac{\lambda x}{\beta R} \\
& \times \exp \left(-\left(\frac{x-2 E\left[|F|_{2}\right]}{\beta R}+\frac{\tilde{\alpha}_{R}^{2}}{\beta^{2} R^{2}}\right) \log \left(1+\frac{\left(x-2 E\left[|F|_{2}\right]\right) \beta R}{\tilde{\alpha}_{R}^{2}}\right)\right) d x \\
\leq & 1+\frac{2}{\beta R} \int_{\beta R / \lambda-2 E|F|_{2}}^{\infty} e^{\frac{u}{\beta R}} e^{\frac{u+2 E\left[|F|_{2}\right]}{\beta R} \log \frac{\lambda\left(u+2 E\left[|F|_{2}\right]\right)}{\beta R}} \\
& \times \exp \left(-\left(\frac{u}{\beta R}+\frac{\tilde{\alpha}_{R}^{2}}{\beta^{2} R^{2}}\right) \log \left(1+\frac{u \beta R}{\tilde{\alpha}_{R}^{2}}\right)\right) \log \frac{\lambda\left(u+2 E\left[|F|_{2}\right]\right)}{\beta R} d u .
\end{aligned}
$$

It then suffices to study the dominant term in the above integral:

$$
\begin{aligned}
& \int_{\beta R / \lambda}^{\infty} \exp \left(\frac{u}{\beta R}-\frac{u}{\beta R} \log \left(1+\frac{u \beta R}{\tilde{\alpha}_{R}^{2}}\right)\right) e^{\frac{u}{\beta R} \log \left(1+\frac{\lambda u}{\beta R}\right)} d u \\
& =\int_{\beta R / \lambda}^{\infty} \exp \left(\frac{u}{\beta R}-\frac{u}{\beta R} \log \frac{1+u \beta R / \tilde{\alpha}_{R}^{2}}{1+\lambda\left(u+2 E\left[|F|_{2}\right]\right) /(\beta R)}\right) d u
\end{aligned}
$$

Since

$$
\lim _{u \rightarrow \infty} \log \frac{1+u \beta R / \tilde{\alpha}_{R}^{2}}{1+\lambda\left(u+2 E\left[|F|_{2}\right]\right) /(\beta R)}=\log \frac{\beta^{2} R^{2}}{\lambda \tilde{\alpha}_{R}^{2}}>1,
$$

for $\lambda<\beta^{2} R^{2} /\left(e \tilde{\alpha}_{R}^{2}\right)$, the convergence of the integral follows from

$$
\int_{\beta R / \lambda}^{\infty} \exp \left(\frac{u}{\beta R}-\frac{u}{\beta R} \log \frac{\beta^{2} R^{2}}{\lambda \tilde{\alpha}_{R}^{2}}\right) d u<\infty .
$$

Since $\tilde{\alpha}_{R}^{2}$ given in Corollary 3.3 does not depend on the dimension, the condition on $\lambda$ in the above corollary is also dimension free.

\section{Random vectors with non-independent components}

First, we obtain the following from Corollary 2.6

Corollary 3.5 Let $f: \mathbb{R}^{n} \rightarrow \mathbb{R}$ be $\ell^{2}$-Lipschitz $(c)$ and let $F=\left(F_{1}, \ldots, F_{n}\right)$ such that $\sum_{j=1}^{n}\left|D_{(i, y)} F_{j}(\omega)\right|^{2} \leq K^{2}, P \otimes \nu_{i}(d \omega, d y)$-a.e., $i=1, \ldots, n$, for some $K \geq 0$ and

$$
\left\|\sum_{i, j=1}^{n}\right\| D_{(i, \cdot)} F_{j}(\omega)\left\|_{L^{2}\left(Y ; \nu_{i}\right)}^{2}\right\|_{L^{\infty}\left(\Omega^{X}, P\right)} \leq \tilde{\alpha}^{2}
$$

Then

$$
P(f(F)-E[f(F)] \geq x) \leq e^{\frac{x}{c K}}\left(1+\frac{x K}{\tilde{\alpha}^{2}}\right)^{-\frac{x}{c K}-\frac{\tilde{\alpha}^{2}}{K^{2}}}, \quad x>0 .
$$


Proof. Note that since $f$ is $\ell^{2}-\operatorname{Lipschitz}(c)$ we have for $G=f(F)$ :

$$
\begin{aligned}
\left(D_{(i, y)} G(\omega)\right)^{2}= & \left(f\left(F_{1}(\omega \cup\{(i, y)\}), \ldots, F_{i}(\omega \cup\{(i, y)\}), \ldots, F_{n}(\omega \cup\{(i, y)\})\right)\right. \\
& \left.-f\left(F_{1}(\omega), \ldots, F_{i}(\omega), \ldots F_{n}(\omega)\right)\right)^{2} \\
\leq & c^{2} \sum_{j=1}^{n}\left|F_{j}(\omega \cup\{(i, y)\})-F_{j}(\omega)\right|^{2} \\
= & c^{2} \sum_{j=1}^{n}\left|D_{(i, y)} F_{j}(\omega)\right|^{2} .
\end{aligned}
$$

So that $\left|D_{(i, y)} G(\omega)\right| \leq c K$ and $\sum_{i=1}^{n}\left\|D_{(i, \cdot)} G(\omega)\right\|_{L^{2}\left(Y ; \nu_{i}\right)}^{2} \leq c^{2} \tilde{\alpha}^{2}, P(d \omega)$-a.s., and Corollary 2.6 applies to $G$.

From Corollary [3.5] we can derive an exponential integrability result for the Euclidean norm of a vector of arbitrary functionals on $X=\{1, \ldots, n\} \times Y$, provided $\nu_{1}=\cdots=\nu_{n}$ has support in $B_{Y}(0, R)$. This completes the sharper result stated in Corollary [3.4 in the case of independent components. However, in the infinitely divisible case, it is slightly less sharp as Corollary 3 of [24].

Corollary 3.6 Let $\nu=\nu_{1}=\cdots=\nu_{n}$ have bounded support in $B_{Y}(0, R)$, and let $F=\left(F_{1}, \ldots, F_{n}\right)$ be a vector of $n$ (non-necessarily independent) random functionals. Assume that

$$
\sum_{j=1}^{n} \frac{\left|D_{(i, y)} F_{j}(\omega)\right|^{2}}{|y|_{Y}^{2}} \leq \tilde{\alpha}^{2}<\infty, \quad P \otimes \nu_{i}(d \omega, d y)-\text { a.e., } i=1, \ldots, n
$$

and

$$
\left\|\sum_{i, j=1}^{n}\right\| D_{(i, y)} F_{j}(\omega)\left\|_{L^{2}\left(Y ; \nu_{i}\right)}^{2}\right\|_{L^{\infty}\left(\Omega^{X}, P\right)} \leq \tilde{\tilde{\alpha}}^{2}<\infty .
$$

Then (13.11) holds for $0<\lambda<\tilde{\alpha}^{2} R^{2} / \tilde{\tilde{\alpha}}^{2}$ :

$$
E\left[\exp \left(\frac{|F|_{2}}{\tilde{\alpha} R} \log _{+} \frac{\lambda^{2}|F|_{2}^{2}}{\tilde{\alpha} R}\right)\right]<\infty .
$$

Proof. First, note that $\left|D_{(i, y)} F_{j}\right| \leq \tilde{\alpha}|y|_{Y} \leq \tilde{\alpha} R$, since $D_{(i, y)} F_{j}$ is zero for $|y|_{Y}>R$, $\nu$ being supported on $B_{Y}(0, R)$. We can thus apply Corollary 3.5 and get

$$
P(f(F)-E[f(F)] \geq x) \leq e^{\frac{x}{\tilde{\alpha} R}}\left(1+\frac{x \tilde{\alpha} R}{\tilde{\alpha}^{2}}\right)^{-\frac{x}{\tilde{\alpha} R}-\frac{\tilde{\tilde{\alpha}}^{2}}{\tilde{\alpha}^{2} R^{2}}}, \quad x>0,
$$


which is (3.12) with $K=\tilde{\alpha} R$ and $c=1$. Finally (3.13) follows from (3.14) as (3.11) follows from (3.10) in Corollary 3.4 .

In the previous corollary, $\tilde{\tilde{\alpha}}$ is dimension dependent, unlike Corollary [3.4, so that the exponential integrability is not dimension free in the dependent case. As an application of Corollary 3.5 we obtain an upper large deviation bound in the dependent case, for random functionals with bounded support.

Corollary 3.7 Let $\nu:=\nu_{1}=\cdots=\nu_{n}$ have bounded support in $B_{Y}(0, R)$ and let $F=\left(F_{1}, \ldots, F_{n}\right)$ be a vector of $n$ (non-necessarily independent) random functionals. Assume that

$$
\sum_{j=1}^{n} \frac{\left|D_{(i, y)} F_{j}(\omega)\right|^{2}}{|y|_{Y}^{2}} \leq \tilde{\alpha}^{2}<\infty, \quad P \otimes \nu_{i}(d \omega, d y)-\text { a.e., } i=1, \ldots, n .
$$

and

$$
\left\|\sum_{i, j=1}^{n}\right\| D_{(i, y)} F_{j}(\omega)\left\|_{L^{2}\left(Y ; \nu_{i}\right)}^{2}\right\|_{L^{\infty}\left(\Omega^{X}, P\right)}<\infty .
$$

Then for any $\ell^{2}$-Lipschitz(c) function $f: \mathbb{R}^{n} \rightarrow \mathbb{R}$, we have

$$
\limsup _{x \rightarrow \infty} \frac{\log P(|f(F)| \geq x)}{x \log x} \leq-\frac{c}{\tilde{\alpha} R} .
$$

When restricted to single Poisson integrals, the previous result recovers the upper estimate of Corollary 4 in [24] in which a deviation result is obtained for the norm of infinitely divisible vector with Lévy measure having a bounded support. See also [12] for related results in the framework of large deviations for Poisson stochastic integrals.

\section{Quadratic Wiener functionals}

The results of the previous section apply in particular to quadratic Wiener functionals since they have infinitely divisible laws, cf. [17, and can be represented as Poisson stochastic integrals with finite variance. Note that exact estimates for the tail probabilities of (quadratic) Wiener functionals have been obtained in [11, see also [5], [13], [18, [19. Here we present dimension free results for norms of vectors of independent quadratic functionals. In this section we take $X=\mathbb{R}$. 


\section{Second order Wiener integrals}

It is well-known (see e.g. [17]) that every centered quadratic Wiener functional can be determined by a symmetric Hilbert-Schmidt operator $A: L^{2}\left(\mathbb{R}_{+}\right) \rightarrow L^{2}\left(\mathbb{R}_{+}\right)$ with eigenvalues $\left(a_{k}\right)_{k \in \mathbb{N}}$ and a complete orthonormal basis of eigenvectors $\left(h_{k}\right)_{k \in \mathbb{N}}$ in $L^{2}\left(\mathbb{R}_{+}\right)$. In particular it can be expressed as a second order Wiener integral $J_{2}\left(f_{2}\right)$ with respect to a standard Brownian motion $\left(B_{t}\right)_{t \in \mathbb{R}_{+}}$, with

$$
J_{2}\left(f_{2}\right)=\frac{1}{2} \sum_{k=0}^{\infty} a_{k}\left(\left(\int_{0}^{\infty} h_{k}(t) d B_{t}\right)^{2}-1\right)
$$

where the series converges in $L^{2}\left(\Omega^{X}\right)$, and $f_{2}$ has the decomposition

$$
f_{2}=\frac{1}{2} \sum_{k=0}^{\infty} a_{k} h_{k} \otimes h_{k}
$$

converging in $L^{2}\left(\mathbb{R}_{+}^{2}\right)$. Note that $J_{2}\left(f_{2}\right)$ is distinct from the double Poisson stochastic integral $I_{2}\left(f_{2}\right)$. The variance of $J_{2}\left(f_{2}\right)$ is

$$
\operatorname{Var}\left[J_{2}\left(f_{2}\right)\right]=\left\|f_{2}\right\|_{L^{2}\left(\mathbb{R}_{+}^{2}\right)}^{2}=\frac{1}{4} \sum_{k=0}^{\infty} a_{k}^{2} .
$$

In the sequel we consider a vector $\left(J_{2}^{1}\left(f_{2}^{1}\right), \ldots, J_{2}^{n}\left(f_{2}^{n}\right)\right)$ of mutually independent second order Wiener integrals of $f_{2}^{1}, \ldots, f_{2}^{n} \in L^{2}\left(\mathbb{R}_{+}^{2}\right)$ with respect to possibly different Brownian motions. Denote also by $\left(a_{k}^{i}\right)_{k \in \mathbb{N}}$ the eigenvalues associated to $J_{2}^{i}\left(f_{2}^{i}\right)$, $i=1, \ldots, n$. For each $i=1, \ldots, n, J_{2}^{i}\left(f_{2}^{i}\right)$ is infinitely divisible, integrable, and centered with Lévy measure

$$
\nu_{i}(d y)=\mathbf{1}_{\{y>0\}} \sum_{k ; a_{k}^{i}>0} \frac{1}{2|y|} e^{-y / a_{k}^{i}} d y+\mathbf{1}_{\{y<0\}} \sum_{k ; a_{k}^{i}<0} \frac{1}{2|y|} e^{-y / a_{k}^{i}} d y,
$$

cf. Theorem 2 of [17]. Hence from (1.3), $J_{2}^{i}\left(f_{2}^{i}\right)$ has the representation

$$
J_{2}^{i}\left(f_{2}^{i}\right)\left(\omega_{i}\right)=\int_{-\infty}^{\infty} y\left(\omega_{i}(d y)-\nu_{i}(d y)\right)
$$

as a single Poisson stochastic integral. Denote by

$$
a_{+}=\max _{1 \leq i \leq n} \max _{k, a_{k}^{i}>0} a_{k}^{i}, \quad a_{-}=\max _{1 \leq i \leq n} \max _{k, a_{k}^{i}<0}\left(-a_{k}^{i}\right), \quad a=\max _{1 \leq i \leq n} \max _{k \in \mathbb{N}}\left|a_{k}^{i}\right|
$$


the maxima of the spectral radii associated to $J_{2}^{1}\left(f_{2}^{1}\right), \ldots, J_{2}^{n}\left(f_{2}^{n}\right)$.

In the next proposition we apply Corollary 2.5 to obtain a deviation result for $\ell^{1}$ Lipschitz functions of quadratic Wiener functionals. Note that Corollary 4 of [9] (or Proposition 3.1 applied to Poisson stochastic integrals) would yield dimension free deviation results when $g$ is $\ell^{2}$-Lipschitz, however with an additional range condition.

Proposition 4.1 Let $\left(J_{2}^{1}\left(f_{2}^{1}\right), \ldots, J_{2}^{n}\left(f_{2}^{n}\right)\right)$ be a vector of independent second order Wiener integrals. For any $\ell^{1}$-Lipschitz(c) function $g$ :

$$
P\left(g\left(J_{2}^{1}\left(f_{2}^{1}\right), \ldots, J_{2}^{n}\left(f_{2}^{n}\right)\right)-E\left[g\left(J_{2}^{1}\left(f_{2}^{1}\right), \ldots, J_{2}^{n}\left(f_{2}^{n}\right)\right)\right] \geq x\right) \leq \exp \left(-\int_{0}^{x} h^{-1}(s) d s\right),
$$

$x>0$, where $h^{-1}$ is the inverse of the function

$$
h(t)=\frac{1}{2} \sum_{i=1}^{n} \sum_{k=0}^{\infty} \frac{c t\left(a_{k}^{i}\right)^{2}}{1-c t\left|a_{k}^{i}\right|}, \quad t \in\left[0,(c a)^{-1}\right) .
$$

Moreover,

$$
\begin{aligned}
& P\left(g\left(J_{2}^{1}\left(f_{2}^{1}\right), \ldots, J_{2}^{n}\left(f_{2}^{n}\right)\right)-E\left[g\left(J_{2}^{1}\left(f_{2}^{1}\right), \ldots, J_{2}^{n}\left(f_{2}^{n}\right)\right)\right] \geq x\right) \\
& \quad \leq \exp \left(-\frac{x}{a c}+\frac{2 \sum_{i=1}^{n}\left\|f_{2}^{i}\right\|_{L^{2}\left(\mathbb{R}_{+}^{2}\right)}^{2}}{a^{2} c} \log \left(1+\frac{a x}{2 \sum_{i=1}^{n}\left\|f_{2}^{i}\right\|_{L^{2}\left(\mathbb{R}_{+}^{2}\right)}^{2}}\right)\right) \\
& \quad \leq \exp \left(-\frac{1}{c}\left(1-\frac{\log 3}{2}\right) \min \left(\frac{x}{a}, \frac{x^{2}}{4 \sum_{i=1}^{n}\left\|f_{2}^{i}\right\|_{L^{2}\left(\mathbb{R}_{+}^{2}\right)}^{2}}\right)\right), \quad x>0 .
\end{aligned}
$$

Proof. From Corollary 2.5 and (4.2), (4.1), (3.4), we have

$$
\begin{aligned}
h(t) & \leq c \sum_{i=1}^{n} \int_{-\infty}^{\infty}|y|\left(e^{t c|y|}-1\right) \nu_{i}(d y) \\
& =\frac{c}{2} \sum_{i=1}^{n} \sum_{\substack{k=0 \\
a_{k}^{i}>0}}^{\infty} \int_{0}^{\infty}\left(e^{t c y}-1\right) e^{-y / a_{k}^{i}} d y+\frac{c}{2} \sum_{i=1}^{n} \sum_{\substack{k=0 \\
a_{k}^{i}<0}}^{\infty} \int_{-\infty}^{0}\left(e^{-t c y}-1\right) e^{-y / a_{k}^{i}} d y \\
& =\frac{1}{2} \sum_{i=1}^{n} \sum_{\substack{k=0 \\
a_{k}^{i}>0}}^{\infty} \frac{c t\left(a_{k}^{i}\right)^{2}}{1-c t a_{k}^{i}}+\frac{1}{2} \sum_{i=1}^{n} \sum_{\substack{k=0 \\
a_{k}^{i}<0}}^{\infty} \frac{c t\left(a_{k}^{i}\right)^{2}}{1+c t a_{k}^{i}} \\
& =\frac{1}{2} \sum_{i=1}^{n} \sum_{k=0}^{\infty} \frac{c t\left(a_{k}^{i}\right)^{2}}{1-c t\left|a_{k}^{i}\right|} .
\end{aligned}
$$


Then one can take

$$
h(t)=\frac{1}{2} \sum_{i=1}^{n} \sum_{k=0}^{\infty} \frac{c t\left(a_{k}^{i}\right)^{2}}{1-c t a} \leq \frac{2 c t}{(1-c t a)} \sum_{i=1}^{n}\left\|f_{2}^{i}\right\|_{L^{2}\left(\mathbb{R}_{+}^{2}\right)}^{2}, \quad t \in\left[0,(c a)^{-1}\right),
$$

and in this case,

$$
h^{-1}(t)=\frac{t}{c a t+2 c \sum_{i=1}^{n}\left\|f_{2}^{i}\right\|_{L^{2}\left(\mathbb{R}_{+}^{2}\right)}^{2}},
$$

from which (4.3) follows with explicit computations.

Alternatively, and since $\lim _{t \rightarrow \infty} h^{-1}(t)=1 /(c a)$, we have for any $\varepsilon>0$

$$
\begin{gathered}
P\left(g\left(J_{2}^{1}\left(f_{2}^{1}\right), \ldots, J_{2}^{n}\left(f_{2}^{n}\right)\right)-E\left[g\left(J_{2}^{1}\left(f_{2}^{1}\right), \ldots, J_{2}^{n}\left(f_{2}^{n}\right)\right)\right] \geq x\right) \\
\leq C_{1}(c, n, \varepsilon) \exp \left(-x\left((c a)^{-1}-\varepsilon\right)\right),
\end{gathered}
$$

$x>0$, for some constant $C_{1}(c, n, \varepsilon)$ depending on $c, n$ and $\varepsilon$. It follows that there is a constant $C_{2}(c, n, \lambda)$ such that

$$
E\left[e^{\lambda\left|g\left(J_{2}^{1}\left(f_{2}^{1}\right), \ldots, J_{2}^{n}\left(f_{2}^{n}\right)\right)\right|}\right]<C_{2}(c, n, \lambda)<\infty,
$$

for all $\lambda<1 /(a c)$, and every $\ell^{1}$-Lipschitz(c) function $g: \mathbb{R}^{n} \rightarrow \mathbb{R}$. In fact, (4.3) implies that for all $\lambda<-1-\frac{2}{a^{2} c} \sum_{i=1}^{n}\left\|f_{2}^{i}\right\|_{L^{2}\left(\mathbb{R}_{+}^{2}\right)}^{2}$,

$E\left[\exp \left(-\frac{1}{a c}\left|g\left(J_{2}^{1}\left(f_{2}^{1}\right), \ldots, J_{2}^{n}\left(f_{2}^{n}\right)\right)\right|+\lambda \log \left(1+\left|g\left(J_{2}^{1}\left(f_{2}^{1}\right), \ldots, J_{2}^{n}\left(f_{2}^{n}\right)\right)\right|\right)\right)\right]<C_{3}(c, n, \lambda)$,

for some $C_{3}(c, n, \lambda)<\infty$. For the supremum of $J_{2}^{1}\left(f_{2}^{1}\right), \ldots, J_{2}^{n}\left(f_{2}^{n}\right)$, which is a Lipschitz function with respect to the $\ell^{\infty}$-norm, hence with respect to the $\ell^{1}$-norm, the previous corollary can be strengthened by making use of (3.5).

Proposition 4.2 Let $\left(J_{2}^{1}\left(f_{2}^{1}\right), \ldots, J_{2}^{n}\left(f_{2}^{n}\right)\right)$ be a vector of independent second order Wiener integrals. Then, $P\left(\sup \left(J_{2}^{1}\left(f_{2}^{1}\right), \ldots, J_{2}^{n}\left(f_{2}^{n}\right)\right)-E\left[\sup \left(J_{2}^{1}\left(f_{2}^{1}\right), \ldots, J_{2}^{n}\left(f_{2}^{n}\right)\right)\right] \geq x\right) \leq \exp \left(-\int_{0}^{x} h^{-1}(s) d s\right)$, $x>0$, where $h^{-1}$ is the inverse of the function

$$
h(t)=\frac{1}{2} \sum_{i=1}^{n} \sum_{\substack{k=0 \\ a_{k}^{i}>0}}^{\infty} \frac{t\left(a_{k}^{i}\right)^{2}}{1-t a_{k}^{i}}, \quad t \in\left[0,1 / a_{+}\right) .
$$


Moreover,

$$
\begin{aligned}
P(\sup ( & \left.\left.J_{2}^{1}\left(f_{2}^{1}\right), \ldots, J_{2}^{n}\left(f_{2}^{n}\right)\right)-E\left[\sup \left(J_{2}^{1}\left(f_{2}^{1}\right), \ldots, J_{2}^{n}\left(f_{2}^{n}\right)\right)\right] \geq x\right) \\
& \leq \exp \left(-\frac{x}{a_{+}}+\frac{2 \sum_{i=1}^{n}\left\|f_{2}^{i}\right\|_{L^{2}\left(\mathbb{R}_{+}^{2}\right)}^{2}}{\left(a_{+}\right)^{2}} \log \left(1+\frac{a_{+} x}{2 \sum_{i=1}^{n}\left\|f_{2}^{i}\right\|_{L^{2}\left(\mathbb{R}_{+}^{2}\right)}^{2}}\right)\right) \\
& \leq \exp \left(-\left(1-\frac{\log 3}{2}\right) \min \left(\frac{x}{a_{+}}, \frac{x^{2}}{4 \sum_{i=1}^{n}\left\|f_{2}^{i}\right\|_{L^{2}\left(\mathbb{R}_{+}^{2}\right)}^{2}}\right)\right), \quad x>0 .
\end{aligned}
$$

Proof. Follow the lines of the proof of Proposition 4.1 starting from (3.6) instead of (3.4).

Note that in dimension one and for second order Wiener integrals, (4.6) above implies the upper deviation bound of [18] (Example 5.1), since

$$
a_{+}=2 \sup _{\|h\|_{L^{2}\left(\mathbb{R}_{+}\right)}=1}\left\langle f_{2}, h \otimes h\right\rangle_{L^{2}\left(\mathbb{R}_{+}^{2}\right)}
$$

Counterparts of (4.4), (4.5) for $\sup \left(J_{2}^{1}\left(f_{2}^{1}\right), \ldots, J_{2}^{n}\left(f_{2}^{n}\right)\right)$ can be derived in the same way. Our next result is a first lower bound.

Proposition 4.3 Let $\left(J_{2}^{1}\left(f_{2}^{1}\right), \ldots, J_{2}^{n}\left(f_{2}^{n}\right)\right)$ be a vector of (centered) mutually independent quadratic Wiener functionals. For any $b \in(0,1)$, there exists $x_{b}>0$ such that

$$
P\left(\left|\left(J_{2}^{1}\left(f_{2}^{1}\right), \ldots, J_{2}^{n}\left(f_{2}^{n}\right)\right)\right|_{\infty} \geq x\right) \geq \frac{1-b}{2 x} \sum_{i=1}^{n} a^{i} e^{-x / a^{i}}, \quad x>x_{b},
$$

with $a^{i}=\max _{k \in \mathbb{N}}\left|a_{k}^{i}\right|, 1 \leq i \leq n$.

Proof. Let $F_{1}, \ldots, F_{n}$ be $n$ independent random variables with respective distribution $I D\left(m_{1}, 0, \nu_{1}\right), \ldots, I D\left(m_{n}, 0, \nu_{n}\right)$. We have for $x>0$ :

$$
\begin{aligned}
P\left(\left|\left(F_{1}, \ldots, F_{n}\right)\right|_{\infty} \geq x\right) & \geq P\left(\exists i \in\{1, \ldots, n\}:\left|F_{i}\right| \geq x\right) \\
& =1-P\left(\left|F_{i}\right|<x, 1 \leq i \leq n\right) \\
& =1-\prod_{i=1}^{n} P\left(\left|F_{i}\right|<x\right) .
\end{aligned}
$$

Writing

$$
F_{i}=F_{i}^{+}+F_{i}^{-}-m_{i}
$$


with

$$
F_{i}^{+}\left(\omega_{i}\right)=\int_{0}^{\infty} y \omega_{i}(d y), \quad F_{i}^{-}\left(\omega_{i}\right)=\int_{-\infty}^{0} y \omega_{i}(d y), \quad m_{i}=\int_{-\infty}^{\infty} y \nu_{i}(d y),
$$

we have

$$
\begin{aligned}
P\left(F_{i}<x\right) & =\int_{0}^{\infty} P\left(F_{i}^{+}<x+m_{i}+y\right) d P\left(F_{i}^{-}=-y\right) \\
& \leq \int_{0}^{\infty} P\left(\omega_{i}\left(\left[x+m_{i}+y, \infty\right)\right)=0\right) d P\left(F_{i}^{-}=-y\right) \\
& =\int_{0}^{\infty} \exp \left(-\nu_{i}\left(\left[x+m_{i}+y, \infty\right)\right)\right) d P\left(F_{i}^{-}=-y\right) .
\end{aligned}
$$

Here,

$$
\nu_{i}([x, \infty))=\int_{x}^{\infty} \sum_{k, a_{k}^{i}>0} \frac{1}{2|y|} e^{-y / a_{k}^{i}} d y \sim_{x \rightarrow \infty} N_{+} \int_{x}^{\infty} \frac{e^{-y / a_{+}^{i}}}{2 y} d y \sim_{x \rightarrow \infty} a_{+}^{i} N_{+}^{i} \frac{e^{-x / a_{+}^{i}}}{2 x},
$$

where $f(x) \sim_{x \rightarrow \infty} g(x)$ means that $\lim _{x \rightarrow+\infty} f(x) / g(x)=1$, and $a_{+}^{i}=\max _{k, a_{k}^{i}>0}\left|a_{k}^{i}\right|$, $N_{+}^{i}=\#\left\{k, a_{k}^{i}=a_{+}^{i}\right\}$. Hence for all $b^{\prime} \in(0,1)$ there exists $x_{i, b^{\prime}}>0$ such that

$$
P\left(F_{i}<x\right) \leq \exp \left(-a_{+}^{i} N_{+}^{i} \frac{e^{-x / a_{+}^{i}}}{2 x}\left(1-b^{\prime}\right)\right), \quad x>x_{i, b^{\prime}} .
$$

Similarly we have

$$
\nu_{i}((-\infty,-x])=\int_{-\infty}^{-x} \sum_{k, a_{k}^{i}<0} \frac{1}{2|y|} e^{-y / a_{k}^{i}} d y \sim_{x \rightarrow \infty} a_{-}^{i} N_{-}^{i} \frac{e^{-x / a_{-}^{i}}}{2 x},
$$

with $a_{-}^{i}=\max _{k, a_{k}^{i}<0}\left|a_{k}^{i}\right|$ and $N_{-}^{i}=\#\left\{k, a_{k}^{i}=-a_{-}^{i}\right\}$. Hence $x_{i, b^{\prime}}$ can be chosen such that

$$
P\left(F_{i}>-x\right) \leq \exp \left(-a_{-}^{i} N_{-}^{i} \frac{e^{-x / a_{-}^{i}}}{2 x}\left(1-b^{\prime}\right)\right), \quad x>x_{i, b^{\prime}},
$$

thus

$$
P\left(\left|F_{i}\right|<x\right) \leq \exp \left(-a^{i} \frac{e^{-x / a^{i}}}{2 x}\left(1-b^{\prime}\right)\right), \quad x>x_{i, b^{\prime}} .
$$

For $x>\max \left\{x_{1, b^{\prime}}, \ldots, x_{n, b^{\prime}}\right\}$. It follows that

$$
P\left(\left|\left(F_{1}, \ldots, F_{n}\right)\right|_{\infty} \geq x\right) \geq 1-\exp \left(-\sum_{i=1}^{n} a^{i} \frac{e^{-x / a^{i}}}{2 x}\left(1-b^{\prime}\right)\right),
$$


and so for any $b \in(0,1)$, there exists $x_{b}>0$ such that

$$
P\left(\left|\left(F_{1}, \ldots, F_{n}\right)\right|_{\infty} \geq x\right) \geq \frac{1-b}{2 x} \sum_{i=1}^{n} a^{i} e^{-x / a^{i}}, \quad x>x_{b} .
$$

Note that without the independence assumption on $\left(J_{2}^{1}\left(f_{2}^{1}\right), \ldots, J_{2}^{n}\left(f_{2}^{n}\right)\right)$, a similar argument leads to the estimate

$$
P\left(\left|\left(J_{2}^{1}\left(f_{2}^{1}\right), \ldots, J_{2}^{n}\left(f_{2}^{n}\right)\right)\right|_{\infty} \geq x\right) \geq a(1-b) \frac{e^{-x / a}}{2 x},
$$

for any $b \in(0,1)$ and $x$ large enough. A version of Proposition 4.3 can also be stated for $\sup \left(J_{2}^{1}\left(f_{2}^{1}\right), \ldots, J_{2}^{n}\left(f_{2}^{n}\right)\right)$. For $n=1$, and for second order Wiener integrals, this also implies the lower deviation bound obtained in Example 5.1 in [18].

Proposition 4.4 Let $\left(J_{2}^{1}\left(f_{2}^{1}\right), \ldots, J_{2}^{n}\left(f_{2}^{n}\right)\right)$ be a vector of independent quadratic Wiener functionals. For any $b \in(0,1)$, there exists $x_{b}>0$ such that

$$
P\left(\sup \left(J_{2}^{1}\left(f_{2}^{1}\right), \ldots, J_{2}^{n}\left(f_{2}^{n}\right)\right) \geq x\right) \geq \frac{1-b}{2 x} \sum_{i=1}^{n} a_{+}^{i} e^{-x / a_{+}^{i}}, \quad x>x_{b},
$$

with $a_{+}^{i}=\max _{k \in \mathbb{N}, a_{k}^{i}>0} a_{k}^{i}, 1 \leq i \leq n$.

Proof. We follow the lines of proof of Proposition 4.3, Let $F_{1}, \ldots, F_{n}$ be $n$ independent random variables with respective distribution $I D\left(m_{1}, 0, \nu_{1}\right), \ldots, I D\left(m_{n}, 0, \nu_{n}\right)$. We have for $x>0$ :

$$
P\left(\sup \left(F_{1}, \ldots, F_{n}\right) \geq x\right) \geq 1-\prod_{i=1}^{n} P\left(F_{i}<x\right),
$$

which leads to

$$
P\left(\sup \left(F_{1}, \ldots, F_{n}\right) \geq x\right) \geq 1-\exp \left(-\sum_{i=1}^{n} a_{+}^{i} \frac{e^{-x / a_{+}^{i}}}{2 x}\left(1-b^{\prime}\right)\right),
$$

for $x$ sufficiently large. Hence, for any $b \in(0,1)$, there exists $x_{b}>0$ such that

$$
P\left(\sup \left(F_{1}, \ldots, F_{n}\right) \geq x\right) \geq \frac{1-b}{2 x} \sum_{i=1}^{n} a_{+}^{i} e^{-x / a_{+}^{i}}, \quad x>x_{b} .
$$


Without the independence assumption on $\left(J_{2}^{1}\left(f_{2}^{1}\right), \ldots, J_{2}^{n}\left(f_{2}^{n}\right)\right)$ we get

$$
P\left(\sup \left(J_{2}^{1}\left(f_{2}^{1}\right), \ldots, J_{2}^{n}\left(f_{2}^{n}\right)\right) \geq x\right) \geq a_{+}(1-b) \frac{e^{-x / a_{+}}}{2 x}
$$

for any $b \in(0,1)$ and $x$ large enough. In the next corollary we derive an exact tail estimate for the $\ell_{p}$-norm of vectors of independent quadratic Wiener functionals, recovering, in the special case of second order integrals, the result obtained in [2] for non-decoupled Gaussian chaos, see also [14, Cor. 3.9].

Corollary 4.5 Let $p \in[1, \infty]$, and let $\left(J_{2}^{1}\left(f_{2}^{1}\right), \ldots, J_{2}^{n}\left(f_{2}^{n}\right)\right)$ be a vector of independent quadratic Wiener functionals. Then

$$
\lim _{x \rightarrow+\infty} \frac{\log P\left(\left|\left(J_{2}^{1}\left(f_{2}^{1}\right), \ldots, J_{2}^{n}\left(f_{2}^{n}\right)\right)\right|_{p} \geq x\right)}{x}=-\frac{1}{a} .
$$

Proof. For any $b \in(0,1)$, from Proposition 4.3 and Proposition 4.1 there exists $x_{b}>0$ such that

$$
\begin{aligned}
& (1-b) \frac{e^{-x / a}}{2 x} \sum_{i=1}^{n} a^{i} \leq P\left(\left|\left(J_{2}^{1}\left(f_{2}^{1}\right), \ldots, J_{2}^{n}\left(f_{2}^{n}\right)\right)\right|_{\infty} \geq x\right) \\
& \leq P\left(\left|\left(J_{2}^{1}\left(f_{2}^{1}\right), \ldots, J_{2}^{n}\left(f_{2}^{n}\right)\right)\right|_{p} \geq x\right) \\
& \quad \leq \exp \left(-\frac{x-M}{a}+\frac{2 \sum_{i=1}^{n}\left\|f_{2}^{i}\right\|_{L^{2}\left(\mathbb{R}_{+}^{2}\right)}^{2}}{a^{2}} \log \left(1+\frac{a(x-M)}{\left.\left.2 \sum_{i=1}^{n}\left\|f_{2}^{i}\right\|_{L^{2}\left(\mathbb{R}_{+}^{2}\right)}^{2}\right)\right),}\right.\right. \\
& x>\max \left(x_{b}, M\right), \text { with } M=E\left[\left|\left(J_{2}^{1}\left(f_{2}^{1}\right), \ldots, J_{2}^{n}\left(f_{2}^{n}\right)\right)\right|_{1}\right] .
\end{aligned}
$$

Note that for $n=1$ and for second order Wiener integrals, the above result coincides with Theorem 2.2 of [5] (see also [13] and [19]), since $a / 2$ is also the strong operator norm of the linear map canonically associated to $f_{2}$, i.e.

$$
a=2 \sup _{\|h\|_{L^{2}\left(\mathbb{R}_{+}\right)}=1}\left|\left\langle f_{2}, h \otimes h\right\rangle_{L^{2}\left(\mathbb{R}_{+}^{2}\right)}\right|
$$

A result of [5] states that

$$
\lim _{x \rightarrow+\infty} \frac{\log P\left(\sup _{t \in \mathbb{R}_{+}}\left|J_{m}\left(f_{m}^{t}\right)\right| \geq x\right)}{x}=-\frac{1}{2 \sup _{t \in \mathbb{R}_{+}}\left\|f_{m}^{t}\right\|_{L^{2}\left(\mathbb{R}_{+}^{m}\right)}},
$$

provided $\left(J_{m}\left(f_{m}^{t}\right)\right)_{t \in \mathbb{R}_{+}}$is a process of $m$-th order integrals with a.s. continuous sample paths (see also Remark 4.3 in [18]). It is clear that for $n=1, m=2$ and $p=+\infty$, (4.8) 
and (4.9) coincide. However, (4.9) does not imply (4.8), since as is well known the process $\left(J_{m}\left(f_{m}^{t}\right)\right)_{t \in \mathbb{R}_{+}}$cannot be jointly measurable and have independent components. For the supremum of $J_{2}^{1}\left(f_{2}^{1}\right), \ldots, J_{2}^{n}\left(f_{2}^{n}\right)$ we similarly have:

Corollary 4.6 Let $\left(J_{2}^{1}\left(f_{2}^{1}\right), \ldots, J_{2}^{n}\left(f_{2}^{n}\right)\right)$ be a vector of independent quadratic Wiener functionals, then

$$
\lim _{x \rightarrow+\infty} \frac{\log P\left(\sup \left(J_{2}^{1}\left(f_{2}^{1}\right), \ldots, J_{2}^{n}\left(f_{2}^{n}\right)\right) \geq x\right)}{x}=-\frac{1}{a_{+}} .
$$

Proof. Apply Proposition 4.2 and Proposition 4.4.

A left deviation estimate for $\sup \left(J_{2}^{1}\left(f_{2}\right), \ldots, J_{2}^{n}\left(f_{2}\right)\right)$ can be independently obtained from

$$
P\left(\sup \left(J_{2}^{1}\left(f_{2}\right), \ldots, J_{2}^{n}\left(f_{2}\right)\right) \leq x\right)=\prod_{k=1}^{n} P\left(J_{2}^{k}\left(f_{2}\right) \leq x\right),
$$

which can then be estimated from Proposition 4.4 or Proposition 9.17 of [1]. Counterparts of (4.6) in Proposition 4.2, as well as Proposition 4.4 Corollary 4.6 and (4.7) can also be stated for the left deviation of $\inf \left(J_{2}^{1}\left(f_{2}\right), \ldots, J_{2}^{n}\left(f_{2}\right)\right)$, replacing $a_{+}^{i}$ by $a_{-}^{i}$, $i=1, \ldots, n$, and $a_{+}$by $a_{-}$. Since

$$
a_{-}=-2 \inf _{\|h\|_{L^{2}\left(\mathbb{R}_{+}\right)}=1}\left\langle f_{2}, h \otimes h\right\rangle_{L^{2}\left(\mathbb{R}_{+}^{2}\right)},
$$

this will imply the one-dimensional left tails of [18] (Example 5.1). For an arbitrary norm $\|\cdot\|$ on $\mathbb{R}^{n}$, we have

$$
\|x\|=\left\|\sum_{i=1}^{i=n} x_{i} e_{i}\right\| \leq|x|_{1} \max _{1 \leq i \leq n}\left\|e_{i}\right\| .
$$

Hence,

$$
\limsup _{x \rightarrow+\infty} \frac{\log P\left(\left\|\left(J_{2}^{1}\left(f_{2}^{1}\right), \ldots, J_{2}^{n}\left(f_{2}^{n}\right)\right)\right\| \geq x\right)}{x} \leq-\frac{1}{a \max _{1 \leq i \leq n}\left\|e_{i}\right\|} .
$$

Similarly, since $\|x\| \geq c(n)|x|_{\infty}$ for some $c(n)>0$,

$$
\liminf _{x \rightarrow+\infty} \frac{\log P\left(\left\|\left(J_{2}^{1}\left(f_{2}^{1}\right), \ldots, J_{2}^{n}\left(f_{2}^{n}\right)\right)\right\| \geq x\right)}{x} \geq-\frac{1}{a c(n)} .
$$

For the Euclidean norm, we also have the following dimension free deviation inequality obtained from Proposition 3.2 for an i.i.d. vector. The independent but non identically distributed case is similar with more notation. 
Proposition 4.7 Let $\left(J_{2}^{1}\left(f_{2}\right), \ldots, J_{2}^{n}\left(f_{2}\right)\right)$ be an i.i.d. vector of second order Wiener integrals, and let $b \in(0,1)$. Then,

$$
P\left(\left|\left(J_{2}^{1}\left(f_{2}\right), \ldots, J_{2}^{n}\left(f_{2}\right)\right)\right|_{2}-2 E\left[\left|\left(J_{2}^{1}\left(f_{2}\right), \ldots, J_{2}^{n}\left(f_{2}\right)\right)\right|_{2}\right] \geq x\right) \leq e^{-(1-b) \frac{x}{a}+K_{b}}, \quad x>0
$$

and

$P\left(\left|\left(J_{2}^{1}\left(f_{2}\right), \ldots, J_{2}^{n}\left(f_{2}\right)\right)\right|_{2}-2 E\left[\left|\left(J_{2}^{1}\left(f_{2}\right), \ldots, J_{2}^{n}\left(f_{2}\right)\right)\right|_{2}\right] \geq x\right) \leq e^{-(1-b) \frac{x}{a}}, \quad x \geq \frac{2 a}{b} K_{b / 2}$,

where

$$
\begin{aligned}
K_{b}= & -\frac{16\left\|f_{2}\right\|_{L^{2}\left(\mathbb{R}_{+}^{2}\right)}^{2}}{a^{2}} \log b-8\left\|f_{2}\right\|_{L^{2}\left(\mathbb{R}_{+}^{2}\right)}^{2}\left(\frac{2}{a^{2}}+\frac{1}{\left(E\left[\left|J_{2}\left(f_{2}\right)\right|\right]\right)^{2}}\right)(1-b) \\
& +\frac{4\left\|f_{2}\right\|_{L^{2}\left(\mathbb{R}_{+}^{2}\right)}^{2}}{\left(E\left[\left|J_{2}\left(f_{2}\right)\right|\right]\right)^{2}}\left(\frac{1-b^{2}}{b^{2}}\right) .
\end{aligned}
$$

Proof. Applying Proposition 3.2 with $\beta=1$ gives

$$
\begin{aligned}
& P\left(\left|\left(J_{2}^{1}\left(f_{2}\right), \ldots, J_{2}^{n}\left(f_{2}\right)\right)\right|_{2}-2 E\left[\left|\left(J_{2}^{1}\left(f_{2}\right), \ldots, J_{2}^{n}\left(f_{2}\right)\right)\right|_{2}\right] \geq x\right) \\
& \quad \leq \exp \left(-\int_{0}^{x} h^{-1}(s) d s\right)=\min _{0<t<1 / a} \exp \left(-t x+\int_{0}^{t} h(s) d s\right), \quad x>0
\end{aligned}
$$

where

$$
\begin{aligned}
h(t)= & 4 \sum_{\substack{k=0 \\
a_{k}>0}}^{\infty} \int_{0}^{\infty}\left(e^{t y}-1\right) e^{-y / a_{k}} d y+\frac{1}{\left(E\left[\left|J_{2}\left(f_{2}\right)\right|\right]\right)^{2}} \sum_{\substack{k=0 \\
a_{k}>0}}^{\infty} \int_{0}^{\infty} y^{2}\left(e^{t y}-1\right) e^{-y / a_{k}} d y \\
& +4 \sum_{\substack{k=0 \\
a_{k}<0}}^{\infty} \int_{-\infty}^{0}\left(e^{-t y}-1\right) e^{-y / a_{k}} d y+\frac{1}{\left(E\left[\left|J_{2}\left(f_{2}\right)\right|\right]\right)^{2}} \sum_{\substack{k=0 \\
a_{k}<0}}^{\infty} \int_{-\infty}^{0} y^{2}\left(e^{-t y}-1\right) e^{-y / a_{k}} d y \\
= & 4 \sum_{\substack{k=0 \\
\leq}}^{\infty} \frac{t a_{k}^{2}}{1-t\left|a_{k}\right|}+\frac{2}{\left(E\left[\left|J_{2}\left(f_{2}\right)\right|\right]\right)^{2}} \sum_{k=0}^{\infty}\left|a_{k}\right|^{3}\left(\frac{1}{\left(1-t\left|a_{k}\right|\right)^{3}}-1\right) \\
\leq & \frac{16\left\|f_{2}\right\|_{L^{2}\left(\mathbb{R}_{+}^{2}\right)}^{2}}{a} \frac{t a}{1-t a}+\frac{8 a\left\|f_{2}\right\|_{L^{2}\left(\mathbb{R}_{+}^{2}\right)}^{2}}{\left(E\left[\left|J_{2}\left(f_{2}\right)\right|\right]\right)^{2}}\left(\frac{1}{(1-t a)^{3}}-1\right) .
\end{aligned}
$$

Letting

$$
A=\frac{16\left\|f_{2}\right\|_{L^{2}\left(\mathbb{R}_{+}^{2}\right)}^{2}}{a^{2}} \quad \text { and } \quad B=\frac{8\left\|f_{2}\right\|_{L^{2}\left(\mathbb{R}_{+}^{2}\right)}^{2}}{\left(E\left[\left|J_{2}\left(f_{2}\right)\right|\right]\right)^{2}}
$$

we have

$$
\int_{0}^{t} h(s) d s \leq-A(\log (1-t a)+t a)+B\left(\frac{1}{2(1-t a)^{2}}-t a-\frac{1}{2}\right)
$$




$$
=-A \log (1-t a)-(A+B) t a+B \frac{t a}{(1-t a)^{2}}-\frac{B}{2} \frac{(t a)^{2}}{(1-t a)^{2}} .
$$

Taking $t=(1-b) / a$, the min in (4.12) is bounded by

$$
\min _{0<t<1 / a} \exp \left(-t x+\int_{0}^{t} h(s) d s\right) \leq \exp \left(-(1-b) \frac{x}{a}+K_{b}\right)
$$

where

$$
K_{b}=\int_{0}^{\frac{1-b}{a}} h(s) d s=-A \log b-(A+B)(1-b)+B \frac{1-b}{b^{2}}-B \frac{(1-b)^{2}}{2 b^{2}},
$$

and (4.10) follows. Taking $t=(1-b / 2) / a$ in (4.12) yields

$$
\min _{0<t<1 / a} \exp \left(-t x+\int_{0}^{t} h(s) d s\right) \leq \exp \left(-(1-b / 2) \frac{x}{a}+K_{b / 2}\right) \leq \exp \left(-(1-b) \frac{x}{a}\right),
$$

$x \geq \frac{2 a}{b} K_{b / 2}$, and (4.11) follows.

Note that the growth of $K_{b}$ is in $1 / b^{2}$, as $b \rightarrow 0$.

\section{Square norm of Brownian paths on $[0, T]$}

An example of quadratic Wiener functional for which the coefficients $\left(a_{k}\right)_{k \in \mathbb{N}}$ can be explicitly computed is given by the (compensated) integrated squared Brownian motion

$$
\mathfrak{h}_{T}=\int_{0}^{T}(B(t))^{2} d t-\frac{T^{2}}{2}
$$

on the interval $[0, T]$. In this case, from [10] or $\S 3.1 .1$ of [17], we have $a_{k}=\frac{4 T^{2}}{(2 k+1)^{2} \pi^{2}}$, $k \geq 0$, and the above results apply with $a=\frac{4 T^{2}}{\pi^{2}}$ and

$$
\sum_{k=0}^{\infty} a_{k}^{2}=\frac{T^{2}}{2} .
$$

Letting $\left(\mathfrak{h}_{T}^{1}, \ldots, \mathfrak{h}_{T}^{n}\right)$ be a vector of i.i.d. copies of $\mathfrak{h}_{T}$, Proposition 4.1 states in this case that for any $\ell^{1}-\operatorname{Lipschitz}(c)$ function $g: \mathbb{R}^{n} \rightarrow \mathbb{R}$ :

$$
\begin{aligned}
P\left(g\left(\mathfrak{h}_{T}^{1}, \ldots, \mathfrak{h}_{T}^{n}\right)-E\left[g\left(\mathfrak{h}_{T}^{1}, \ldots, \mathfrak{h}_{T}^{n}\right)\right] \geq x\right) \\
\quad \leq \exp \left(-\frac{\pi^{2} x}{4 c T^{2}}+\frac{n \pi^{4}}{32 T^{2} c} \log \left(1+\frac{8 x}{n \pi^{2}}\right)\right)
\end{aligned}
$$




$$
\leq \exp \left(-\left(1-\frac{\log 3}{2}\right) \min \left(\frac{\pi^{2} x}{4 c T^{2}}, \frac{x^{2}}{n c T^{2}}\right)\right), \quad x>0
$$

and for all $\varepsilon>0$ :

$$
P\left(g\left(\mathfrak{h}_{T}^{1}, \ldots, \mathfrak{h}_{T}^{n}\right)-E\left[g\left(\mathfrak{h}_{T}^{1}, \ldots, \mathfrak{h}_{T}^{n}\right)\right] \geq x\right) \leq C_{1}(c, n, T, \varepsilon) \exp \left(-x\left(\pi^{2} /\left(4 c T^{2}\right)-\varepsilon\right)\right)
$$

$x>0$. It follows that

$$
E\left[e^{\lambda\left|g\left(\mathfrak{h}_{T}^{1}, \ldots, \mathfrak{h}_{T}^{n}\right)\right|}\right]<C_{2}(c, n, T, \lambda),
$$

for all $\lambda<\pi^{2} /\left(4 c T^{2}\right)$ and some constant $C_{2}(c, n, T, \lambda)<\infty$. For any $b \in(0,1)$ we also get the lower bound

$$
P\left(\left|\left(\mathfrak{h}_{T}^{1}, \ldots, \mathfrak{h}_{T}^{n}\right)\right|_{\infty} \geq x\right) \geq n(1-b) \frac{\pi^{2}}{8 x T^{2}} e^{-x \pi^{2} /\left(4 T^{2}\right)}
$$

for $x$ sufficiently large, and from Corollary 4.5 .

$$
\lim _{x \rightarrow+\infty} \frac{\log P\left(\left|\left(\mathfrak{h}_{T}^{1}, \ldots, \mathfrak{h}_{T}^{n}\right)\right|_{p} \geq x\right)}{x}=-\frac{\pi^{2}}{4 T^{2}}, \quad p \in[1, \infty] .
$$

For the Euclidean norm and from Proposition 4.7, we again have dimension free deviation inequalities: for any $b \in(0,1)$,

$$
P\left(\left|\left(\mathfrak{h}_{T}^{1}, \ldots, \mathfrak{h}_{T}^{n}\right)\right|_{2}-2 E\left[\left|\left(\mathfrak{h}_{T}^{1}, \ldots, \mathfrak{h}_{T}^{n}\right)\right|_{2}\right] \geq x\right) \leq \exp \left(-\frac{\pi^{2}(1-b)}{4 T^{2}} x+K_{b}\right), \quad x \geq 0
$$

and

$P\left(\left|\left(\mathfrak{h}_{T}^{1}, \ldots, \mathfrak{h}_{T}^{n}\right)\right|_{2}-2 E\left[\left|\left(\mathfrak{h}_{T}^{1}, \ldots, \mathfrak{h}_{T}^{n}\right)\right|_{2}\right] \geq x\right) \leq \exp \left(-\frac{\pi^{2}(1-b)}{4 T^{2}} x\right), \quad x \geq \frac{8 T^{2}}{\pi^{2} b} K_{b / 2}$

where

$$
K_{b}=-\frac{\pi^{4}}{8 T^{2}} \log b-T^{2}\left(\frac{\pi^{4}}{8 T^{4}}+\frac{1}{\left(E\left[\left|\mathfrak{h}_{T}\right|\right]\right)^{2}}\right)(1-b)+\frac{T^{2}}{2\left(E\left[\left|\mathfrak{h}_{T}\right|\right]\right)^{2}} \frac{1-b^{2}}{b^{2}} .
$$

\section{Sample variance of Brownian motion on $[0, T]$}

A second example is given by the (compensated) sample variance of Brownian motion

$$
\mathfrak{v}_{T}=\int_{0}^{T}\left(B(t)-\frac{1}{T} \int_{0}^{T} B(s) d s\right)^{2} d t-\frac{T^{2}}{6}
$$


on the interval $[0, T]$. From [10], or $\S 3.3 .1$ of [17], $a_{k}=\frac{T^{2}}{k^{2} \pi^{2}}, k \geq 1, a_{0}=0, a=\frac{T^{2}}{\pi^{2}}$ and

$$
\sum_{k=1}^{\infty} a_{k}^{2}=\frac{T^{2}}{6}
$$

Letting $\left(\mathfrak{v}_{T}^{1}, \ldots, \mathfrak{v}_{T}^{n}\right)$ be a vector of i.i.d. copies of $\mathfrak{v}_{T}$, we have from Proposition 4.1 that for every $\ell^{1}$-Lipschitz $(c)$ function $g: \mathbb{R}^{n} \rightarrow \mathbb{R}$ :

$$
\begin{aligned}
P\left(g\left(\mathfrak{v}_{T}^{1}, \ldots, \mathfrak{v}_{T}^{n}\right)-E\left[g\left(\mathfrak{v}_{T}^{1}, \ldots, \mathfrak{v}_{T}^{n}\right)\right] \geq x\right) & \\
& \leq \exp \left(-\frac{\pi^{2} x}{c T^{2}}+\frac{n \pi^{4}}{6 T^{2} c} \log \left(1+\frac{6 x}{n \pi^{2}}\right)\right) \\
& \leq \exp \left(-\left(1-\frac{\log 3}{2}\right) \min \left(\frac{\pi^{2} x}{c T^{2}}, \frac{6 x^{2}}{2 n c T^{2}}\right)\right), \quad x>0
\end{aligned}
$$

and for all $\varepsilon>0$ :

$$
P\left(g\left(\mathfrak{v}_{T}^{1}, \ldots, \mathfrak{v}_{T}^{n}\right)-E\left[g\left(\mathfrak{v}_{T}^{1}, \ldots, \mathfrak{v}_{T}^{n}\right)\right] \geq x\right) \leq C_{1}(c, n, T, \varepsilon) \exp \left(-x\left(\pi^{2} /\left(c T^{2}\right)-\varepsilon\right)\right)
$$

$x>0$. It follows that

$$
E\left[e^{\lambda\left|g\left(\mathfrak{v}_{T}^{1}, \ldots, \mathfrak{v}_{T}^{n}\right)\right|}\right]<C_{2}(c, n, T, \lambda),
$$

for all $\lambda<\pi^{2} /\left(c T^{2}\right)$ and some $C_{2}(c, n, T, \lambda)<\infty$. For any $b \in(0,1)$ we also get the lower bound

$$
P\left(\left|\left(\mathfrak{v}_{T}^{1}, \ldots, \mathfrak{v}_{T}^{n}\right)\right|_{\infty} \geq x\right) \geq n(1-b) \frac{\pi^{2}}{2 x T^{2}} e^{-x \pi^{2} / T^{2}},
$$

for $x$ sufficiently large, and from Corollary 4.5

$$
\lim _{x \rightarrow+\infty} \frac{\log P\left(\left|\left(\mathfrak{v}_{T}^{1}, \ldots, \mathfrak{v}_{T}^{n}\right)\right|_{p} \geq x\right)}{x}=-\frac{\pi^{2}}{T^{2}}, \quad p \in[1, \infty] .
$$

From Proposition 4.7 for any $b \in(0,1)$ :

$$
P\left(\left|\left(\mathfrak{v}_{T}^{1}, \ldots, \mathfrak{v}_{T}^{n}\right)\right|_{2}-2 E\left[\left|\left(\mathfrak{v}_{T}^{1}, \ldots, \mathfrak{v}_{T}^{n}\right)\right|_{2}\right] \geq x\right) \leq \exp \left(-\frac{\pi^{2}(1-b)}{T^{2}} x+K_{b}\right), \quad x \geq 0,
$$

and

$$
P\left(\left|\left(\mathfrak{v}_{T}^{1}, \ldots, \mathfrak{v}_{T}^{n}\right)\right|_{2}-2 E\left[\left|\left(\mathfrak{v}_{T}^{1}, \ldots, \mathfrak{v}_{T}^{n}\right)\right|_{2}\right] \geq x\right) \leq \exp \left(-\frac{\pi^{2}(1-b)}{T^{2}} x\right), \quad x \geq \frac{2 T^{2}}{\pi^{2} b} K_{b / 2},
$$

where

$$
K_{b}=-\frac{2}{3} \frac{\pi^{4}}{T^{2}} \log b-\frac{T^{2}}{3}\left(\frac{2 \pi^{4}}{T^{4}}+\frac{1}{\left(E\left[\left|\mathfrak{v}_{T}\right|\right]\right)^{2}}\right)(1-b)+\frac{T^{2}}{6\left(E\left[\left|\mathfrak{v}_{T}\right|\right]\right)^{2}} \frac{1-b^{2}}{b^{2}} .
$$




\section{Lévy's stochastic area}

Let $\left(B^{1}(t), B^{2}(t)\right)_{t \in \mathbb{R}_{+}}$, be a two-dimensional Brownian motion. Lévy's stochastic area $S_{T}$ on $[0, T]$ is

$$
S_{T}=\frac{1}{2} \int_{0}^{T}\left(B^{1}(t) d B^{2}(t)-B^{2}(t) d B^{1}(t)\right),
$$

cf. [15]. For $S_{T}$, the expression of the coefficients $\left(a_{k}\right)_{k \in \mathbb{N}}$ is intricate (see [17]), hence, we can not directly specialize the results of Proposition 4.1, 4.7 and Corollary 4.5] in that case. However, since the Lévy measure of $S_{T}$ has the analytic expression

$$
\nu(d y)=\frac{1}{2 y \sinh \frac{\pi y}{T}} d y
$$

(cf. page 175 of [15], §3.2.1 of [17] or Example 15.15 of [25]), we can derive results similar to the ones obtained for general second order Wiener-Itô integrals.

Proposition 4.8 Let $g: \mathbb{R}^{n} \rightarrow \mathbb{R}$ be $\ell^{1}$-Lipschitz $(c)$, and let $\left(S_{T}^{1}, \ldots, S_{T}^{n}\right)$ be an i.i.d. vector of Lévy's stochastic areas on $[0, T]$. Then,

$$
P\left(g\left(S_{T}^{1}, \ldots, S_{T}^{n}\right)-E\left[g\left(S_{T}^{1}, \ldots, S_{T}^{n}\right)\right] \geq x\right) \leq\left(1+\frac{\pi x}{4 n c T}\right)^{4 n} \exp \left(-\frac{\pi x}{c T}\right) .
$$

Proof. Using the representation of $S_{T}$ as the compensated Poisson stochastic integral

$$
\int_{-\infty}^{\infty} y(\omega(d y)-\nu(d y))
$$

and (3.4) derived from Corollary 2.5, we have

$$
\begin{aligned}
h(t) & =n c \int_{-\infty}^{\infty}|y|_{Y}\left(e^{t c|y|_{Y}}-1\right) \nu(d y) \\
& =n c \int_{-\infty}^{\infty}|y|_{Y}\left(e^{t c|y|_{Y}}-1\right) \frac{1}{2 y \sinh \frac{\pi y}{T}} d y \\
& =2 n c \int_{0}^{\infty} \frac{e^{t c y}-1}{e^{\pi y / T}-e^{-\pi y / T}} d y \\
& \leq 2 n c \int_{0}^{\infty} \frac{e^{t c y}-1}{e^{\pi y / T}-1} d y \\
& \leq \frac{2 n c^{2} t T}{\pi} \int_{0}^{\infty} e^{-\frac{y}{2}\left(\frac{\pi}{T}-c t\right)} d y \\
& \leq \frac{4 n c^{2} t T}{\pi\left(\frac{\pi}{T}-c t\right)}, \quad 0<t<\pi /(c T),
\end{aligned}
$$


using the inequality

$$
\frac{e^{u x}-1}{e^{v x}-1} \leq \frac{u}{v} e^{(u-v) x / 2}, \quad x>0,
$$

for $0<u<v$. Hence

$$
-\int_{0}^{x} h^{-1}(s) d s \leq-\frac{\pi^{2}}{T} \int_{0}^{x} \frac{s}{4 n c^{2} T+c \pi s} d s=-\frac{\pi}{c T} x+4 n \log \left(1+\frac{\pi x}{4 n c T}\right) .
$$

The above result can also be obtained from Theorem 1 in [6] in place of (3.4). Alternatively, we have $\lim _{t \rightarrow \infty} h^{-1}(t)=\pi(c T)^{-1}$ since $\lim _{t \rightarrow \pi(c T)^{-1}} h(t)=+\infty$. Hence for all $\varepsilon>0$, we also derive as in (4.4)

$$
P\left(g\left(S_{T}^{1}, \ldots, S_{T}^{n}\right)-E\left[g\left(S_{T}^{1}, \ldots, S_{T}^{n}\right)\right] \geq x\right) \leq C_{1}(c, n, T, \varepsilon) \exp \left(-x\left(\pi(c T)^{-1}-\varepsilon\right)\right),
$$

$x>0$, for some constant $C_{1}(c, n, T, \varepsilon)$ depending on $T, c, n$ and $\varepsilon$. This last inequality is not dimension free. Nevertheless it yields

$$
E\left[e^{\lambda\left|g\left(S_{T}^{1}, \ldots, S_{T}^{n}\right)\right|}\right]<C_{2}(c, n, T, \lambda)<\infty,
$$

for all $\lambda<\pi /(c T)$, and every $\ell^{1}$-Lipschitz $(c)$ function $g: \mathbb{R}^{n} \rightarrow \mathbb{R}$.

Proposition 4.9 Let $p \geq 1$. For all $b \in(0,1)$ there exists $x_{b}>0$ such that

$$
P\left(\left|\left(S_{T}^{1}, \ldots, S_{T}^{n}\right)\right|_{\infty} \geq x\right) \geq(1-b) \frac{n T e^{-\pi x / T}}{2 \pi x}, \quad x>x_{b} .
$$

Proof. Given $n$ i.i.d. random variables $F_{1}, \ldots, F_{n}$ with distribution $I D(m, 0, \nu)$, we have from the proof of Proposition 4.3 .

$$
P\left(\left|\left(F_{1}, \ldots, F_{n}\right)\right|_{\infty} \geq x\right) \geq 1-\left(P\left(\left|F_{1}\right|<x\right)\right)^{n},
$$

while for $\nu$ given in (4.13) we have the equivalence:

$$
\nu\left(\left[x, \infty[)=\int_{x}^{\infty} \frac{d y}{2 y \sinh \frac{\pi y}{T}} \sim_{x \rightarrow \infty} \int_{x}^{\infty} \frac{e^{-\pi y / T}}{y} d y \sim_{x \rightarrow \infty} \frac{T e^{-\pi x / T}}{\pi x},\right.\right.
$$

hence for all $b^{\prime} \in(0,1)$ there exists $x_{i, b^{\prime}}$ such that

$$
P\left(\left|F_{i}\right|<x\right) \leq \exp \left(-\left(1-b^{\prime}\right) \frac{T e^{-\pi x / T}}{2 \pi x}\right), \quad x>x_{i, b^{\prime}}, \quad 1 \leq i \leq n .
$$

Thus for $x$ large enough,

$$
P\left(\left|\left(S_{T}^{1}, \ldots, S_{T}^{n}\right)\right|_{\infty} \geq x\right) \geq 1-\exp \left(-(1-b) \frac{n T e^{-\pi x / T}}{2 \pi x}\right) .
$$


The corollary below is a direct consequence of Proposition 4.8 and Proposition 4.9, It recovers a univariate result of [3] and extends it to $\ell^{p}$-norms of i.i.d. random vectors, independently of their dimension. For non identical variables $S_{T_{1}}^{1}, \ldots, S_{T_{n}}^{n}$, replace $T$ by $\max _{1 \leq k \leq n} T_{k}$.

Corollary 4.10 Let $p \in[1, \infty]$. Then,

$$
\lim _{x \rightarrow+\infty} \frac{\log P\left(\left|\left(S_{T}^{1}, \ldots, S_{T}^{n}\right)\right|_{p} \geq x\right)}{x}=-\frac{\pi}{T} .
$$

Note that from the above results we have $a=T / \pi$ and $\sum_{k=0}^{\infty} a_{k}^{2}=T^{2} / 4$. Moreover as a consequence of Proposition 3.2, we have:

Proposition 4.11 Let $|\cdot|_{2}$ denote the Euclidean norm on $\mathbb{R}^{n}$ and let $b \in(0,1)$. We have

$$
P\left(\left|\left(S_{T}^{1}, \ldots, S_{T}^{n}\right)\right|_{2}-2 E\left[\left|\left(S_{T}^{1}, \ldots, S_{T}^{n}\right)\right|_{2}\right] \geq x\right) \leq \exp \left(-\frac{(1-b) \pi}{T} x+K_{b}\right), \quad x>0
$$

and

$$
P\left(\left|\left(S_{T}^{1}, \ldots, S_{T}^{n}\right)\right|_{2}-2 E\left[\left|\left(S_{T}^{1}, \ldots, S_{T}^{n}\right)\right|_{2}\right] \geq x\right) \leq \exp \left(-\frac{(1-b) \pi}{T} x\right), \quad x \geq \frac{2 T}{\pi b} K_{b / 2}
$$

where

$$
K_{b}=-32 \log b-32(1-b)+\frac{16 T^{2}}{\pi^{2}\left(E\left[\left|S_{T}^{1}\right|\right]\right)^{2}} \frac{(1-b)^{2}}{b^{2}} .
$$

Proof. Since for $F$ given in (4.14), $\beta=1$, from Proposition 3.2 and (2.6), we have

$$
P\left(\left|\left(S_{T}^{1}, \ldots, S_{T}^{n}\right)\right|_{2}-2 E\left[\left|\left(S_{T}^{1}, \ldots, S_{T}^{n}\right)\right|_{2}\right] \geq x\right) \leq \exp \left(-t x+\int_{0}^{t} h(s) d s\right),
$$

for all $x>0$ and $0<t<\pi / T$. From (4.15) we have

$$
\begin{aligned}
h(t) & =16 \int_{0}^{\infty} e^{t y / 2} \frac{\sinh \frac{t y}{2}}{\sinh \frac{\pi y}{T}} d y+\frac{4}{\left(E\left[\left|S_{T}^{1}\right|\right]\right)^{2}} \int_{0}^{\infty} y^{2} e^{t y / 2} \frac{\sinh \frac{t y}{2}}{\sinh \frac{\pi y}{T}} d y \\
& \leq \frac{16 t T}{\pi} \int_{0}^{\infty} e^{-\frac{y}{2}\left(\frac{\pi}{T}-t\right)} d y+\frac{4 t T}{\pi\left(E\left[\left|S_{T}^{1}\right|\right]\right)^{2}} \int_{0}^{\infty} y^{2} e^{-y\left(\frac{\pi}{T}-t\right) / 2} d y \\
& \leq \frac{32 T}{\pi} \frac{(t T / \pi)}{1-(t T / \pi)}+\frac{32 T^{3}}{\pi^{3}\left(E\left[\left|S_{T}^{1}\right|\right]\right)^{2}} \frac{(t T / \pi)}{(1-(t T / \pi))^{3}},
\end{aligned}
$$


hence

$$
\begin{aligned}
& P\left(\left|\left(S_{T}^{1}, \ldots, S_{T}^{n}\right)\right|_{2}-2 E\left[\left|\left(S_{T}^{1}, \ldots, S_{T}^{n}\right)\right|_{2}\right] \geq x\right) \\
& \quad \leq \exp \left(-t x-32((t T / \pi)+\log (1-(t T / \pi)))+\frac{16 T^{2}}{\pi^{2}\left(E\left[\left|S_{T}^{1}\right|\right]\right)^{2}} \frac{(t T / \pi)^{2}}{(1-(t T / \pi))^{2}}\right) .
\end{aligned}
$$

For all $b \in(0,1)$, (4.17) follows by taking $t=(1-b) \pi / T$ in (4.19), and (4.18) is a consequence of (4.17) where $b$ is replaced with $b / 2$.

\section{The infinite variance case}

In [7], deviation results have been derived for Lipschitz functions of stable random vectors. In this section, we extend these results to general Poisson functionals under arbitrary intensity measures. Deviations are now given with respect to a median rather than to the mean (which may not exist). For $A$ in $\mathcal{B}(X)$ (the Borel $\sigma$-field of $X$ ), let $\nu_{R}(A)=\nu\left(A \cap B_{X}(0, R)\right)$, where 0 denotes an arbitrary fixed point in $X$. The proofs of the forthcoming results are inspired by that of Theorem 1 in [7]: configurations are truncated and we will use the following notation on the configuration space. For a fixed $R>0$ and any $\omega \in \Omega^{X}$, let

$$
\omega_{R}=\omega \cap B_{X}(0, R), \quad \omega_{R}^{c}=\omega \cap B_{X}(0, R)^{c}=\left\{x \in \omega: d_{X}(0, x)>R\right\} .
$$

Given a stochastic functional $F$ on the configuration space, we also set

$$
F_{R}(\omega)=F\left(\omega_{R}\right)=F\left(\omega \cap B_{X}(0, R)\right),
$$

and denote by $\gamma$ a non-negative and non-increasing function such that

$$
P\left(\left\{\omega \in \Omega^{X}: \omega \cap B_{X}(0, R)^{c} \neq \emptyset\right\}\right) \leq \gamma(R)
$$

for all $R$ large enough. The next Lemma will be used in the sequel. It allows to control $m\left(F_{R}\right)-m(F)$ as in [7].

Lemma 5.1 Let $F$ be a stochastic functional on the configuration space such that there exists a non-negative and non-decreasing function $\tilde{\beta}$ (resp. non-increasing function $\tilde{\gamma}$ ) defined on $\mathbb{R}_{+}$, such that for all $R$ greater than a given $R_{0}$ :

$$
P\left(F_{R}-m\left(F_{R}\right) \geq \tilde{\beta}(R)\right) \leq \tilde{\gamma}(R) \text {. }
$$


Then we have

$$
m\left(F_{R}\right)-m(F) \leq \tilde{\beta}(R),
$$

for all $R$ such that

$$
R \geq \max \left(R_{0}, \inf _{0<\delta<1 / 2} \max \left(\gamma^{-1}(\delta), \tilde{\gamma}^{-1}\left(\frac{1}{2}-\delta\right)\right)\right) .
$$

Proof. The case $m(F) \geq m\left(F_{R}\right)$ being trivial, we consider henceforth $m\left(F_{R}\right) \geq m(F)$. Let $0<\delta<1 / 2$ and assume

$$
R \geq \gamma^{-1}(\delta)
$$

We have

$$
\begin{aligned}
0<\frac{1}{2}-\delta & \leq \frac{1}{2}-\gamma(R) \\
& \leq P(F \leq m(F))-P\left(\left\{\omega \in \Omega^{X}: \omega_{R}^{c} \neq \emptyset\right\}\right) \\
& =E\left[\mathbf{1}_{\left\{F\left(\omega_{R} \cup \omega_{R}^{c}\right) \leq m(F)\right\}}-\mathbf{1}_{\left\{\omega_{R}^{c} \neq \emptyset\right\}}\right] \\
& \leq E\left[\mathbf{1}_{\left\{F\left(\omega_{R}\right) \leq m(F)\right\}}\right] \\
& =P\left(F_{R} \leq m(F)\right) \\
& =P\left(F_{R}-m\left(F_{R}\right) \leq m(F)-m\left(F_{R}\right)\right) \\
& =P\left(-F_{R}-m\left(-F_{R}\right) \geq m\left(F_{R}\right)-m(F)\right),
\end{aligned}
$$

where we used the fact that $-m\left(F_{R}\right)$ is a median of $-F_{R}$. Consider the decreasing function

$$
H_{R}(x)=P\left(-F_{R}-m\left(-F_{R}\right) \geq x\right), \quad x \in \mathbb{R},
$$

and let $I_{R}(y)=\sup \left\{z \geq 0, H_{R}(z) \geq y\right\}$ denote its inverse. We have

$$
m\left(F_{R}\right)-m(F) \leq I_{R}\left(P(F \leq m(F))-P\left(\left\{\omega \in \Omega^{X}: \omega_{R}^{c} \neq \emptyset\right\}\right)\right) \leq I_{R}\left(\frac{1}{2}-\delta\right) .
$$

Assume further that

$$
R \geq \tilde{\gamma}^{-1}\left(\frac{1}{2}-\delta\right)
$$

From (5.1) applied to $-F_{R}$, we have

$$
H_{R}(\tilde{\beta}(R))=P\left(-F_{R}-m\left(-F_{R}\right) \geq \tilde{\beta}(R)\right) \leq \tilde{\gamma}(R) \leq \frac{1}{2}-\delta,
$$


that is finally $I_{R}\left(\frac{1}{2}-\delta\right) \leq \tilde{\beta}(R)$, and from (5.4):

$$
m\left(F_{R}\right)-m(F) \leq I_{R}\left(\frac{1}{2}-\delta\right) \leq \tilde{\beta}(R) .
$$

The next result provides a general deviation property for stochastic functionals with infinite variance on Poisson space.

Theorem 5.2 Let $F$ be a stochastic functional on the configuration space such that there exists a non-negative and non-decreasing function $\beta$, defined on $\mathbb{R}_{+}$, and a constant $C>0$ such that for all $R$ greater than a given $R_{0}$ :

(i) $\sup _{y \in B_{X}(0, R)}\left|D_{y} F(\omega)\right| \leq \beta(R), \quad P(d \omega)$-a.s.,

(ii) $\|D F\|_{L^{\infty}\left(\Omega^{X}, L^{2}\left(\nu_{R}\right)\right)}^{2} \leq C \beta^{2}(R) \gamma(R)$.

Then

$$
P(F-m(F) \geq x) \leq(1+C e) \gamma \circ \beta^{-1}(x / 4)
$$

for all

$$
x \geq 2 \beta\left(\gamma^{-1}\left(\frac{1}{2(1+C e)}\right)\right) .
$$

Proof. Configurations are truncated to deal on the one hand with the functional restricted to the truncated configuration and on the other hand with the rest of the configuration which is controlled using the function $\gamma$. We have

$$
\begin{aligned}
P(F-m(F) \geq x) & =P\left(F-m(F) \geq x, \omega_{R}^{c}=\emptyset\right)+P\left(F-m(F) \geq x, \omega_{R}^{c} \neq \emptyset\right) \\
& \leq P\left(F_{R}-m(F) \geq x\right)+P\left(\left\{\omega \in \Omega^{X}: \omega_{R}^{c} \neq \emptyset\right\}\right)
\end{aligned}
$$

For the first term, in order to apply (2.10) in Corollary 2.4 (which provides a deviation result from the mean rather than from a median), let

$$
g(x)=\left(x-m\left(F_{R}\right)\right)^{+} \wedge r, \quad x \in \mathbb{R} .
$$

Then $E\left[g\left(F_{R}\right)\right] \leq r P\left(F_{R} \geq m\left(F_{R}\right)\right) \leq r / 2$. Moreover if $F_{R} \geq m\left(F_{R}\right)+r$ then $g\left(F_{R}\right) \geq g\left(m\left(F_{R}\right)+r\right) \geq r$, hence

$$
\left\{F_{R} \geq m\left(F_{R}\right)+r\right\} \subset\left\{g\left(F_{R}\right) \geq r\right\}
$$


and

$$
P\left(F_{R}-m\left(F_{R}\right) \geq r\right) \leq P\left(g\left(F_{R}\right) \geq r\right) \leq P\left(g\left(F_{R}\right)-E\left[g\left(F_{R}\right)\right] \geq r / 2\right) .
$$

On the other hand, $g\left(F_{R}\right)$ satisfies

$$
D_{y} g\left(F_{R}\right)(\omega) \leq\left|g\left(F_{R}(\omega \cup\{y\})\right)-g\left(F_{R}(\omega)\right)\right| \leq\left|F_{R}(\omega \cup\{y\})-F_{R}(\omega)\right|=\left|D_{y} F\left(\omega_{R}\right)\right|,
$$

since $g: \mathbb{R} \rightarrow \mathbb{R}$ is Lipschitz(1). Thus

$$
\sup _{y \in B_{X}(0, R)} D_{y} g\left(F_{R}\right) \leq \beta(R) \quad \text { and } \quad\left\|D g\left(F_{R}\right)\right\|_{L^{\infty}\left(\Omega^{X}, L^{2}\left(\nu_{R}\right)\right)}^{2} \leq C \gamma(R) \beta(R)^{2},
$$

and from (5.6) and Corollary 2.4 we get

$$
P\left(F_{R}-m\left(F_{R}\right) \geq x\right) \leq e^{x /(2 \beta(R))}\left(1+\frac{x}{2 C \gamma(R) \beta(R)}\right)^{-x /(2 \beta(R))},
$$

and taking $x=2 \beta(R)$ we have:

$$
P\left(F_{R}-m\left(F_{R}\right) \geq 2 \beta(R)\right) \leq e\left(1+\frac{1}{C \gamma(R)}\right)^{-1} \leq e C \gamma(R),
$$

and from Lemma 5.1 with $\tilde{\beta}(R)=2 \beta(R), \tilde{\gamma}(R)=C e \gamma(R)$ and condition (5.1) given by (5.7), we get:

$$
m\left(F_{R}\right)-m(F) \leq 2 \beta(R)
$$

i.e. using (5.7):

$$
P\left(F_{R}-m(F) \geq 4 \beta(R)\right) \leq P\left(F_{R}-m\left(F_{R}\right) \geq 2 \beta(R)\right) \leq C e \gamma(R),
$$

i.e. for $x \geq 4 \beta(R)$, we have

$$
P\left(F_{R}-m(F) \geq x\right) \leq C e \gamma \circ \beta^{-1}\left(\frac{x}{4}\right),
$$

under condition (5.3) which can be rewritten in terms of $x$ as

$$
x \geq \max \left(2 \beta\left(\gamma^{-1}(\delta)\right), 2 \beta\left(\gamma^{-1}\left(\frac{1}{C e}\left(\frac{1}{2}-\delta\right)\right)\right)\right),
$$

i.e

$$
\gamma \circ \beta^{-1}(x / 2) \leq \min \left(\delta, \frac{1}{C e}\left(\frac{1}{2}-\delta\right)\right) .
$$


The optimal bound with $\delta \in(0,1 / 2)$, being obtained for $\delta_{0}=\frac{1}{2(1+C e)} \in(0,1 / 2)$, i.e. the condition on $x$ becomes

$$
x \geq 2 \beta \circ \gamma^{-1}\left(\frac{1}{2(1+C e)}\right) .
$$

The estimate (5.8), together with

$$
P\left(\left\{\omega \in \Omega^{X}: \omega_{R}^{c} \neq \emptyset\right\}\right) \leq \gamma(R) \leq \gamma \circ \beta^{-1}\left(\frac{x}{4}\right),
$$

gives

$$
P(F-m(F) \geq x) \leq(C e+1) \gamma \circ \beta^{-1}\left(\frac{x}{4}\right),
$$

using (5.5), under the condition (5.9).

Note that in the hypotheses of Theorem 5.2 it is sufficient to assume

$$
\sup _{y \in B_{X}(0, R)}\left|D_{y} F\left(\omega_{R}\right)\right| \leq \beta(R), \quad P(d \omega) \text {-a.s. }
$$

and

$$
\left\|D F_{R}\right\|_{L^{\infty}\left(\Omega^{X}, L^{2}\left(\nu_{R}\right)\right)}^{2} \leq C \beta^{2}(R) \gamma(R),
$$

instead of $(i)$ and (ii). The next corollary presents a particular and more tractable case of Theorem 5.2 .

Corollary 5.3 Let $F: \Omega^{X} \rightarrow \mathbb{R}$, and let

$$
\gamma(R)=1-e^{-\nu\left(\left\{y \in X: d_{X}(0, y)>R\right\}\right)}, \quad R>0,
$$

and assume that

$$
\sup _{y \in B_{X}(0, R) \leq R}\left|D_{y} F\right| \leq C^{\prime} R \quad \text { and } \quad\|D F\|_{L^{\infty}\left(\Omega^{X}, L^{2}\left(\nu_{R}\right)\right)}^{2} \leq C R^{2} \gamma(R),
$$

for all $R \geq R_{0}>0$. Then

$$
P(F-m(F) \geq x) \leq\left(1+\frac{C e}{\left(C^{\prime}\right)^{2}}\right) \gamma\left(\frac{x}{4 C^{\prime}}\right), \quad x \geq 2 C^{\prime} \gamma^{-1}\left(\frac{1}{2\left(1+e C /\left(C^{\prime}\right)^{2}\right)}\right) .
$$


On $\mathbb{R}^{n}$ equipped with the Euclidean norm $|\cdot|_{2}$, consider an $\ell^{2}$-Lipschitz $(c)$ function $f: \mathbb{R}^{n} \longrightarrow \mathbb{R}$ and a $n$-dimensional infinitely divisible random vector $F=\left(F_{1}, \ldots, F_{n}\right)$ without Gaussian component and with Lévy measure $\nu$. Let us apply Corollary 5.3 to the random functional $G=f(F)$, where $F$ is given as in (1.3) by:

$$
F=\left(\int_{\left\{|y|_{2} \leq 1\right\}} y_{k}(\omega(d y)-\nu(d y))+\int_{\left\{|y|_{2}>1\right\}} y_{k} \omega(d y)+b_{k}\right)_{1 \leq k \leq n} .
$$

For the gradient, we have if $y \notin \omega$ :

$$
\begin{aligned}
\left|D_{y} G(\omega)\right| & =|G(\omega \cup\{y\})-G(\omega)| \\
& =\left|f\left(\int_{\left\{|u|_{2} \leq 1\right\}} u(\omega(d u)-\nu(d u))+\int_{\left\{|u|_{2}>1\right\}} \omega(d u)+y+b\right)-f(F)\right| \\
& \leq c|y|_{2},
\end{aligned}
$$

since $f$ is $\ell^{2}$-Lipschitz $(c)$, and we obtain $\left|D_{y} G(\omega)\right| \leq c R$, for $|y|_{2} \leq R$. In this case, for $G=f(F)$ the conclusion of Corollary 5.3 reads

$$
P(G-m(G) \geq x) \leq(1+C e)\left(1-\exp \left(-\nu\left(\left\{u \in \mathbb{R}^{n}:|u|_{2}>\frac{x}{4 c}\right\}\right)\right)\right)
$$

When $f(x)=|x|_{2}$ is the Euclidean norm on $\mathbb{R}^{n}$, Lemma 5.4 below also yields a lower bound on $P\left(|F-m|_{2} \geq x\right)$ which has the same order as the upper bound (5.10).

Lemma 5.4 Let $F$ be an infinitely divisible random vector $I D(b, 0, \nu)$ in $\mathbb{R}^{n}$, with median $m \in \mathbb{R}^{n}$. Then

$$
P(\|F-m\| \geq x) \geq \frac{1}{4}\left(1-\exp \left(-\nu\left(\left\{u \in \mathbb{R}^{n}:\|u\| \geq 2 x\right\}\right)\right)\right), \quad x>0,
$$

where $\|\cdot\|$ denotes any norm on $\mathbb{R}^{n}$.

Proof. We start by assuming that $F$ is symmetric with median 0 . Then, since $F$ can be taken to be the value $F(1)$ at time 1 of a Lévy process $(F(t))_{0 \leq t \leq 1}$ starting from $F(0)=0$, we have from Lévy's inequality:

$$
\begin{aligned}
P(\|F\| \geq x) & =P\left(\left\|\sum_{k=1}^{n} F\left(\frac{k}{n}\right)-F\left(\frac{k-1}{n}\right)\right\| \geq x\right) \\
& \geq \frac{1}{2} P\left(\max _{1 \leq j \leq n}\left\|F\left(\frac{j}{n}\right)-F\left(\frac{j-1}{n}\right)\right\| \geq x\right) .
\end{aligned}
$$


Hence

$$
\begin{aligned}
P(\|F\| \geq x) & \geq \liminf _{n \rightarrow \infty} \frac{1}{2} P\left(\max _{1 \leq j \leq n}\left\|F\left(\frac{j}{n}\right)-F\left(\frac{j-1}{n}\right)\right\| \geq x\right) \\
& \geq \frac{1}{2} P\left(\liminf _{n \rightarrow \infty} \max _{1 \leq j \leq n}\left\|F\left(\frac{j}{n}\right)-F\left(\frac{j-1}{n}\right)\right\|>x\right) \\
& \geq \frac{1}{2} P\left(\max _{s \in[0,1]}\left\|F(s)-F\left(s^{-}\right)\right\|>x\right) \\
& \geq \frac{1}{2}\left(1-\exp \left(-\nu\left\{u \in \mathbb{R}^{n}:\|u\| \geq x\right\}\right)\right),
\end{aligned}
$$

where (5.12) is a $n$-dimensional extension of Ex. 22.1 in [25], which relies on the fact that if $\omega$ on $\mathbb{R}_{+} \times \mathbb{R}^{n}$ has a jump of $\|\cdot\|$-norm greater than $x$, then $\max _{s \in[0,1]} \| F(s)-$ $F\left(s^{-}\right) \|>x$. In the general case where $F$ is not necessarily symmetric we apply the above to $F-G$, where $G$ denotes an independent copy of $F$, and use the inequality

$$
P(\|F-m\| \geq x)=\frac{1}{2} P(\|F-m\| \geq x)+\frac{1}{2} P(\|G-m\| \geq x) \geq \frac{1}{2} P(\|F-G\| \geq 2 x) .
$$

We now present several examples of Lévy measures $\nu$ for which the function $\gamma$ can be explicitly computed, and where $F$ has infinite variance, i.e. $\int_{\mathbb{R}^{n}}\|y\|^{2} \nu(d y)=\infty$, but where $f(F)$ satisfies the above hypothesis for $f$ an $\ell^{2}$-Lipschitz $(c)$ function.

1. Let $\mathbb{R}^{n} \backslash\{0\}$ be equipped with the measure given for $B \in \mathcal{B}\left(\mathbb{R}^{n} \backslash\{0\}\right)$ by

$$
\nu(B)=\int_{S^{n-1}} \sigma(d \xi) \int_{0}^{\infty} \mathbf{1}_{B}(r \xi) \frac{|\log r|}{r^{2}} d r,
$$

where $\sigma$ is again a spherical finite measure. Since

$$
\int_{\left\{|y|_{2} \leq 1\right\}}|y|_{2}^{2} \nu(d y)=\sigma\left(S^{n-1}\right)<\infty
$$

and

$$
\nu\left(\left\{|x|_{2} \geq 1\right\}\right)=\int_{S^{n-1}} \sigma(d \xi) \int_{1}^{\infty} \frac{|\log r|}{r^{2}} d r<\infty,
$$

$\nu$ is a Lévy measure. Moreover $\int_{\left\{|y|_{2} \geq 1\right\}}|y|_{2}^{2} \nu(d y)=\infty$, hence $F$ has infinite variance. As before:

$$
P\left(\left\{\omega \in \Omega^{X}: \omega_{R}^{c} \neq \emptyset\right\}\right)=1-\exp \left(\int_{S^{n-1}} \sigma(d \xi) \int_{\left\{|r \xi|_{2} \geq R\right\}} \frac{|\log r|}{r^{2}} d r\right)
$$




$$
\begin{aligned}
& =1-\exp \left(-\sigma\left(S^{n-1}\right) \int_{R}^{\infty} \frac{\log r}{r^{2}} d r\right) \\
& \leq \sigma\left(S^{n-1}\right) \frac{1+\log R}{R}, \quad R>1 .
\end{aligned}
$$

Thus, choose $\gamma(R)=2 \sigma\left(S^{n-1}\right) \frac{\log R}{R}$. On the other hand,

$$
\begin{aligned}
\| D f(F) & \|_{L^{\infty}\left(\Omega^{X}, L^{2}\left(\nu_{R}\right)\right)}^{2} \leq \int_{\left\{|y|_{2} \leq R\right\}} c^{2}|y|_{2}^{2} \nu(d y) \\
& =-c^{2} \int_{S^{n-1}} \sigma(d \xi) \int_{0}^{1} \log r d r+c^{2} \int_{S^{n-1}} \sigma(d \xi) \int_{1}^{R} \log r d r \\
& =c^{2} \sigma\left(S^{n-1}\right)(R \log R-R+2) \\
& \leq c^{2} R^{2} \gamma(R) / 2 .
\end{aligned}
$$

2. Let $X=\mathbb{R}^{n}$, with the finite measure $\nu$ given for $B \in \mathcal{B}\left(\mathbb{R}^{n}\right)$ by

$$
\nu(B)=\int_{S^{n-1}} \sigma(d \xi) \int_{0}^{\infty} \mathbf{1}_{B}(r \xi) \frac{e^{-1 /\left(2 r^{2}\right)}}{r^{2} \sqrt{2 \pi}} d r .
$$

We have

$$
\int_{\left\{|y|_{2} \leq 1\right\}}|y|_{2}^{2} \nu(d y)=\sigma\left(S^{n-1}\right) \int_{0}^{1} \frac{e^{-1 /\left(2 r^{2}\right)}}{\sqrt{2 \pi}} d r<\infty,
$$

so that $\nu$ is a Lévy measure. The infinitely divisible random variable given by the Poisson stochastic integral in (1.3) is thus another example of a random variable without finite variance since

$$
\int_{\left\{|y|_{2} \geq 1\right\}}|y|_{2}^{2} \nu(d y)=\infty
$$

Once more:

$$
\begin{aligned}
P\left(\omega_{R}^{c} \neq \emptyset\right) & =1-\exp \left(-\int_{S^{n-1}} \sigma(d \xi) \int_{\left\{|r \xi|_{2} \geq R\right\}} \frac{e^{-1 /\left(2 r^{2}\right)}}{r^{2} \sqrt{2 \pi}} d r\right) \\
& \leq \sigma\left(S^{n-1}\right) \int_{R}^{\infty} \frac{e^{-1 /\left(2 r^{2}\right)}}{r^{2} \sqrt{2 \pi}} d r \\
& =\sigma\left(S^{n-1}\right) \int_{0}^{1 / R} \frac{e^{-u^{2} / 2}}{\sqrt{2 \pi}} d u .
\end{aligned}
$$

Choose $\gamma(R)=\frac{\sigma\left(S^{n-1}\right)}{\sqrt{2 \pi} R}$. Moreover,

$$
\|D f(F)\|_{L^{\infty}\left(\Omega^{X}, L^{2}\left(\nu_{R}\right)\right)}^{2} \leq c^{2} \int_{B(0, R)}|y|_{2}^{2} \nu_{R}(d y)
$$




$$
\begin{aligned}
& =c^{2} \int_{S^{n-1}} \sigma(d \xi) \int_{0}^{R} r^{2} \frac{e^{-1 /\left(2 r^{2}\right)}}{r^{2} \sqrt{2 \pi}} d r \\
& \leq c^{2} \sigma\left(S^{n-1}\right) \int_{0}^{R} \frac{e^{-1 /\left(2 r^{2}\right)}}{\sqrt{2 \pi}} d r \\
& =c^{2} \sigma\left(S^{n-1}\right) \int_{1 / R}^{\infty} \frac{e^{-u^{2} / 2}}{\sqrt{2 \pi}} \frac{d u}{u^{2}} \\
& \leq c^{2} \sigma\left(S^{n-1}\right)\left(\frac{R e^{-1 /\left(2 R^{2}\right)}}{\sqrt{2 \pi}}-\int_{1 / R}^{\infty} \frac{e^{-u^{2} / 2}}{\sqrt{2 \pi}} d u\right) \\
& \leq c^{2} R^{2} \gamma(R) .
\end{aligned}
$$

3. The above deviation results for $f(F)$ with $F$ as in (1.3) a stable or an infinitely divisible random variable, and with Lévy measure either given by (6.1) or (5.13) or (5.14), continue to hold after minor changes for Hölder continuous functions of order $0<h<1$. Indeed, for such a function $f$ we have

$$
\left|D_{y} f(F)\right|_{2} \leq c|y|_{2}^{h} \leq c R^{h}, \quad|y|_{2} \leq R
$$

For instance in the case of the Lévy measure (5.13) we have

$$
\begin{aligned}
\|D f(F)\|_{L^{\infty}\left(\Omega^{X}, L^{2}\left(\nu_{R}\right)\right)}^{2} \leq \int_{S^{n-1}} \sigma(d \xi) \int_{0}^{R} c^{2} r^{2 h} \nu_{R}(d r) \\
\quad=c^{2} \sigma\left(S^{n-1}\right) \int_{0}^{R} r^{2 h-2}|\log r| d r \\
\quad=-\frac{c^{2} \sigma\left(S^{n-1}\right)}{2} \int_{0}^{1} r^{2 h-2} \log r d r+c^{2} \int_{1}^{R} r^{2 h-2} \log r d r \\
\leq c^{2} \sigma\left(S^{n-1}\right)\left(\frac{R^{2 h-1} \log R}{2 h-1}-\frac{R^{2 h-1}}{(2 h-1)^{2}}+\frac{1}{(2 h-1)^{2}}+\frac{1}{2 h+1}\right) .
\end{aligned}
$$

We can thus apply Theorem 5.2 to $G=f(F)$ with (up to multiplicative constants) the functions:

$$
\beta(R)=R^{h}, \quad \text { and } \quad \gamma(R)=\frac{\log R}{R} .
$$

A similar computation yields in the case of the Lévy measure (5.14):

$$
\|D F\|_{L^{\infty}\left(\Omega^{X}, L^{2}\left(\nu_{R}\right)\right)}^{2} \leq \int_{\left\{|x|_{2} \leq R\right\}} c^{2}|x|_{2}^{2 h} \nu_{R}(d r)
$$




$$
\begin{aligned}
& \leq \frac{c^{2}}{\sqrt{2 \pi}} \int_{S^{n-1}} \sigma(d \xi) \int_{0}^{R} r^{2 h-2} e^{-1 /\left(2 r^{2}\right)} d r \\
& \leq c^{2} \sigma\left(S^{n-1}\right) \frac{R^{2 h-1} e^{-1 /\left(2 R^{2}\right)}}{(2 h-1) \sqrt{2 \pi}} .
\end{aligned}
$$

Once more, Theorem 5.2 applies here, in the Hölder continuous case, with up to multiplicative constants,

$$
\beta(R)=R^{h}, \quad \text { and } \quad \gamma(R)=\frac{e^{-1 /\left(2 R^{2}\right)}}{R \sqrt{2 \pi}}
$$

Before turning to the case of stable intensity measures in the next section, we prove the following lemma for a general intensity measure $\nu$, which is a generalization of Lemma 2 in [7].

Lemma 5.5 Let $F: \Omega^{X} \longrightarrow \mathbb{R}$ and $\alpha_{2}, \alpha_{3}, \alpha_{4}, K>0$, such that

(i) $\sup _{y \in X}\left|D_{y} F(\omega)\right| \leq K<\infty, P(d \omega)-a . s$.

(ii) $\|D F\|_{L^{\infty}\left(\Omega^{X}, L^{k}(\nu)\right)}^{k} \leq \alpha_{k}<\infty, k=2,3,4$.

Assume moreover $\alpha_{3} \leq 2 \alpha_{4} / K$ and $K^{2} \alpha_{2} / \alpha_{4} \geq 2$. Let $s_{0}$ be the (unique) positive solution of

$$
s\left(\alpha_{2}-\frac{\alpha_{4}}{K^{2}}\right)=\frac{\alpha_{4}}{K^{3}}\left(e^{s K}-1\right) .
$$

Let $x_{0}=3 s_{0}\left(\alpha_{2}-\alpha_{4} / K^{2}\right)$. Then for all $x \leq x_{0}$,

$$
P(F-E[F] \geq x) \leq \exp \left(-\frac{x^{2}}{6\left(\alpha_{2}-\alpha_{4} / K^{2}\right)}\right)
$$

while for $x \geq x_{0}$,

$$
P(F-E[F] \geq x) \leq K_{0} \exp \left(\frac{x}{K}-\left(\frac{x}{K}+\frac{3 \alpha_{4}}{K^{4}}\right) \log \left(1+\frac{K^{3} x}{3 \alpha_{4}}\right)\right)
$$

with

$$
K_{0}=\exp \left(-\frac{x_{0}}{K}+\left(\frac{x_{0}}{K}+\frac{3 \alpha_{4}}{K^{4}}\right) \log \left(1+\frac{K^{3} x_{0}}{3 \alpha_{4}}\right)-\frac{x_{0}^{2}}{6\left(\alpha_{2}-\alpha_{4} / K^{2}\right)}\right) .
$$


Proof. From Proposition 2.2 we have

$$
P(F-E[F] \geq x) \leq \exp \left(-\int_{0}^{x} h^{-1}(s) d s\right), \quad 0<x<h\left(t_{0}^{-}\right)
$$

with $h$ given in (2.2). Using the bounds $\left|D_{y} F\right| \leq K$ and

$$
e^{s u}-1 \leq s u+\frac{s^{2}}{2} u^{2}+\frac{e^{s K}-1-s K-s^{2} K^{2} / 2}{K^{3}} u^{3}, \quad 0 \leq u \leq K, s \geq 0,
$$

we have

$$
\begin{aligned}
h(s) \leq & \sup _{\omega, \omega^{\prime} \in \Omega_{X}} \int_{X}\left(s\left|D_{y} F(\omega)\right|\left|D_{y} F\left(\omega^{\prime}\right)\right|+\frac{s^{2}}{2}\left|D_{y} F(\omega)\right|^{2}\left|D_{y} F\left(\omega^{\prime}\right)\right|\right. \\
& \left.+\frac{e^{s K}-1-s K-s^{2} K^{2} / 2}{K^{3}}\left|D_{y} F(\omega)\right|^{3}\left|D_{y} F\left(\omega^{\prime}\right)\right|\right) \nu(d y) \\
\leq & s \sup _{\omega, \omega^{\prime} \in \Omega_{X}} \int_{X}\left|D_{y} F(\omega)\right|\left|D_{y} F\left(\omega^{\prime}\right)\right| \nu(d y)+\frac{s^{2}}{2} \sup _{\omega, \omega^{\prime} \in \Omega_{X}} \int_{X}\left|D_{y} F(\omega)\right|^{2}\left|D_{y} F\left(\omega^{\prime}\right)\right| \nu(d y) \\
& +\frac{e^{s K}-1-s K-s^{2} K^{2} / 2}{K^{3}} \sup _{\omega, \omega^{\prime} \in \Omega_{X}} \int_{X}\left|D_{y} F(\omega)\right|^{3}\left|D_{y} F\left(\omega^{\prime}\right)\right| \nu(d y) .
\end{aligned}
$$

Using the inequality $x y \leq x^{p} / p+y^{q} / q$ for $p^{-1}+q^{-1}=1$ and $x, y \geq 0$, we have for $p=q=2$ :

$$
\begin{aligned}
& \sup _{\omega, \omega^{\prime} \in \Omega_{X}} \int_{X}\left|D_{y} F(\omega)\right|\left|D_{y} F\left(\omega^{\prime}\right)\right| \nu(d y) \\
& \leq \frac{1}{2} \sup _{\omega, \omega^{\prime} \in \Omega_{X}} \int_{X}\left|D_{y} F(\omega)\right|^{2} \nu(d y)+\frac{1}{2} \sup _{\omega, \omega^{\prime} \in \Omega_{X}} \int_{X}\left|D_{y} F\left(\omega^{\prime}\right)\right|^{2} \nu(d y) \leq \alpha_{2},
\end{aligned}
$$

for $q=3$ :

$$
\begin{aligned}
& \sup _{\omega, \omega^{\prime} \in \Omega_{X}} \int_{X}\left|D_{y} F(\omega)\right|^{2}\left|D_{y} F\left(\omega^{\prime}\right)\right| \nu(d y) \\
& \leq \frac{2}{3} \sup _{\omega, \omega^{\prime} \in \Omega_{X}} \int_{X}\left|D_{y} F(\omega)\right|^{3} \nu(d y)+\frac{1}{3} \sup _{\omega, \omega^{\prime} \in \Omega_{X}} \int_{X}\left|D_{y} F\left(\omega^{\prime}\right)\right|^{3} \nu(d y) \leq \alpha_{3},
\end{aligned}
$$

and similarly for $q=4$ :

$$
\begin{aligned}
& \sup _{\omega, \omega^{\prime} \in \Omega_{X}} \int_{X}\left|D_{y} F(\omega)\right|^{3}\left|D_{y} F\left(\omega^{\prime}\right)\right| \nu(d y) \\
& \leq \frac{3}{4} \sup _{\omega, \omega^{\prime} \in \Omega_{X}} \int_{X}\left|D_{y} F(\omega)\right|^{4} \nu(d y)+\frac{1}{4} \sup _{\omega, \omega^{\prime} \in \Omega_{X}} \int_{X}\left|D_{y} F\left(\omega^{\prime}\right)\right|^{4} \nu(d y) \leq \alpha_{4} .
\end{aligned}
$$


From (5.21) we get

$$
\begin{aligned}
h(s) & \leq s \alpha_{2}+\frac{s^{2}}{2} \alpha_{3}+\frac{e^{s K}-1-s K-\frac{s^{2}}{2} K^{2}}{K^{3}} \alpha_{4} \\
& =s\left(\alpha_{2}-\frac{\alpha_{4}}{K^{2}}\right)+\frac{s^{2}}{2}\left(\alpha_{3}-\frac{\alpha_{4}}{K}\right)+\frac{\alpha_{4}}{K^{3}}\left(e^{s K}-1\right) .
\end{aligned}
$$

Since we assume $\alpha_{3} \leq 2 \alpha_{4} / K$, the second summand in the right-hand side of (5.22) is bounded by the third one for all $s \geq 0$. We may now end the proof as in Lemma 2 of [7]:

$$
\begin{aligned}
h(s) & \leq 3 \max \left(s\left(\alpha_{2}-\frac{\alpha_{4}}{K^{2}}\right), \frac{s^{2}}{2}\left(\alpha_{3}-\frac{\alpha_{4}}{K}\right), \frac{\alpha_{4}}{K^{3}}\left(e^{s K}-1\right)\right) \\
& =3 \max \left(s\left(\alpha_{2}-\frac{\alpha_{4}}{K^{2}}\right), \frac{\alpha_{4}}{K^{3}}\left(e^{s K}-1\right)\right) \\
& \leq \begin{cases}3 s\left(\alpha_{2}-\frac{\alpha_{4}}{K^{2}}\right), & 0 \leq s \leq s_{0}, \\
3 \frac{\alpha_{4}}{K^{3}}\left(e^{s K}-1\right), & s \geq s_{0},\end{cases}
\end{aligned}
$$

where $s_{0}$ is the unique positive solution of (5.15) which is well defined since $K^{2} \alpha_{2} / \alpha_{4} \geq 2$. Hence, for $x_{0}=3 s_{0}\left(\alpha_{2}-\alpha_{4} / K^{2}\right)$,

$$
h^{-1}(t)= \begin{cases}\frac{t}{3\left(\alpha_{2}-\alpha_{4} / K^{2}\right)} & \text { for } 0 \leq t \leq x_{0}, \\ \frac{1}{K} \log \left(1+\frac{K^{3}}{3 \alpha_{4}} t\right) & \text { for } t \geq x_{0},\end{cases}
$$

which yields (5.16) and (5.17) from (5.19).

Lemma 5.5 will be used in the proof of Theorem 6.1 below to obtain a deviation result under $\alpha$-stable Lévy measures for all value of $\alpha \in(0,2)$. The following lemma applies only for $\alpha \geq 1$, but will yield a slightly better range condition in Theorem 6.2. and is stated without boundedness assumption on 4th the order moment.

Lemma 5.6 Let $F: \Omega^{X} \longrightarrow \mathbb{R}$ and $\alpha_{2}, \alpha_{3}, K>0$, such that $K \alpha_{2} \geq 2 \alpha_{3}$ and

(i) $\|D F\|_{L^{\infty}\left(\Omega^{X}, L^{2}(\nu)\right)}^{2} \leq \alpha_{2}<\infty$,

(ii) $\|D F\|_{L^{\infty}\left(\Omega^{x}, L^{3}(\nu)\right)}^{3} \leq \alpha_{3}<\infty$,

(iii) $\sup _{y \in X}\left|D_{y} F(\omega)\right| \leq K<\infty, P(d \omega)$-a.s. 
Denote by $s_{0}$ the unique solution of

$$
\frac{e^{s K}-1}{s K}=K \frac{\alpha_{2}}{\alpha_{3}}-1 .
$$

Let also $x_{0}=2 s_{0}\left(\alpha_{2}-\alpha_{3} / K\right)$. Then

$$
P(F-E[F] \geq x) \leq \exp \left(-\frac{x^{2}}{4\left(\alpha_{2}-\alpha_{3} / K\right)}\right), \quad 0 \leq x \leq x_{0},
$$

and

$$
P(F-E[F] \geq x) \leq K_{0} \exp \left(\frac{x}{K}-\left(\frac{x}{K}+2 \frac{\alpha_{3}}{K^{3}}\right) \log \left(1+\frac{K^{2} x}{2 \alpha_{3}}\right)\right), \quad x \geq x_{0},
$$

with

$$
K_{0}=\exp \left(-x_{0} / K+\left(\frac{x_{0}}{K}+\frac{2 \alpha_{3}}{K^{3}}\right) \log \left(1+\frac{K^{2} x_{0}}{2 \alpha_{3}}\right)-\frac{x_{0}^{2}}{4\left(\alpha_{2}-\alpha_{3} / K\right)}\right) .
$$

Proof. As in the proof of Lemma 5.5. apply Proposition 2.2 with $h$ given in (2.2) and bounded by

$$
h(s) \leq s \alpha_{2}+\frac{e^{s K}-1-s K}{K^{2}} \alpha_{3} .
$$

using

$$
e^{s u}-1 \leq s u+\frac{e^{s K}-1-s K}{K^{2}} u^{2}, \quad u \in[0, K],
$$

instead of (5.20). We get

$$
h(s) \leq 2 \max \left(s\left(\alpha_{2}+\frac{\alpha_{3}}{K}\right), \frac{e^{s K}-1}{K^{2}} \alpha_{3}\right) \leq\left\{\begin{array}{cc}
2 s\left(\alpha_{2}-\alpha_{3} / K\right), & s \leq s_{0} \\
2\left(e^{s K}-1\right) \alpha_{3} / K^{2}, & s \geq s_{0}
\end{array}\right.
$$

which allows to conclude as in the proof of Lemma 5.5

\section{The case of stable Lévy measures}

Let $0<\alpha<2, X=\mathbb{R}^{n}$ and the stable Lévy measure given by

$$
\nu(B)=\int_{S^{n-1}} \sigma(d \xi) \int_{0}^{\infty} \mathbf{1}_{B}(r \xi) r^{-1-\alpha} d r, \quad B \in \mathcal{B}\left(\mathbb{R}^{n}\right),
$$

where $\sigma$ is a finite positive measure on $S^{n-1}$, the unit sphere of $\mathbb{R}^{n}$, called the spherical component of $\nu$. We have

$$
P\left(\left\{\omega \in \Omega^{X}: \omega_{R}^{c} \neq \emptyset\right\}\right)=1-P\left(\left\{\omega \in \Omega^{X}: \omega_{R}^{c}=\emptyset\right\}\right)
$$




$$
\begin{aligned}
& =1-\exp \left(-\int_{\left\{|y|_{2}>R\right\}} \nu(d y)\right) \\
& =1-\exp \left(\int_{S^{n-1}} \sigma(d \xi) \int_{\left\{|r \xi|_{2} \geq R\right\}} \frac{d r}{r^{1+\alpha}}\right) \\
& =1-\exp \left(-\frac{\sigma\left(S^{n-1}\right)}{\alpha} R^{-\alpha}\right) \\
& \leq \frac{\sigma\left(S^{n-1}\right)}{\alpha R^{\alpha}}
\end{aligned}
$$

Thus we can take

$$
\gamma(R)=\frac{\sigma\left(S^{n-1}\right)}{\alpha R^{\alpha}}, \quad R>0 .
$$

in Theorem 5.2. Let $f: \mathbb{R}^{n} \rightarrow \mathbb{R}$ be $\ell^{2}$-Lipschitz $(c)$. In case $F$ is a stable random variable represented by a single Poisson stochastic integral of the form (1.3), we have from (2.13):

$$
\begin{aligned}
\|D f(F)\|_{L^{\infty}\left(\Omega^{X}, L^{2}\left(\nu_{R}\right)\right)}^{2} & \leq \int_{\left\{|y|_{2} \leq R\right\}} c^{2}|y|_{2}^{2} \nu(d y) \\
& =c^{2} \int_{S^{n-1}} \sigma(d \xi) \int_{\left\{|r \xi|_{2} \geq R\right\}} r^{1-\alpha} d r \\
& \leq \frac{c^{2} \sigma\left(S^{n-1}\right)}{2-\alpha} R^{2-\alpha} \\
& \leq \frac{2 c^{2}}{2-\alpha} R^{2} \gamma(R),
\end{aligned}
$$

hence Theorem 1 of [7] is recovered taking $\beta(r)=c r$ and $C=2 /(2-\alpha)$ in Theorem 5.2, i.e.

$$
P(f(F)-m(f(F)) \geq x) \leq\left(1+\frac{2 e}{2-\alpha}\right) \frac{\sigma\left(S^{n-1}\right)}{\alpha}\left(\frac{x}{4 c}\right)^{-\alpha}
$$

for all $x$ such that

$$
x \geq 2 c \gamma^{-1}(2-\alpha) \geq 2 c \gamma^{-1}\left(\frac{1}{2(1+2 e /(2-\alpha))}\right),
$$

where $F$ is a stable random variable with parameter $\alpha$. The constant in front of $x^{-\alpha}$ in (6.3) explodes as $\alpha$ goes to 0 or to 2. In fact, as noted in [7], the dependency in $\alpha^{-1}$ of the constant is sharp as $\alpha$ goes to 0 (take for example a symmetric $\alpha$-stable random variable). This explosion does not occur however when $\alpha$ goes to 2 , and the aim of the next result is to provide a deviation bound with such a non-exploding constant, 
for general random variables on Poisson space under $\alpha$-stable intensity measures. The proof relies on Lemma 5.5, and in the particular case of stable random variables, this result also improves Theorem 2 of [7] by allowing $\alpha$ to be arbitrary.

Theorem 6.1 Let $\alpha \in(0,2)$ and $F: \Omega^{X} \rightarrow \mathbb{R}$ such that

$$
\left|D_{y} F(\omega)\right| \leq c|y|_{X}, \quad P(d \omega) \otimes \nu(d y)-\text { a.e. }
$$

with $c>0$. Then we have

$$
P(F-m(F) \geq x) \leq \sigma\left(S^{n-1}\right)\left(\frac{3}{2} e^{2}+\frac{1}{\alpha}\right) \frac{(4 c)^{\alpha}}{x^{\alpha}}
$$

for all

$x \geq 4 c \sigma\left(S^{n-1}\right)^{1 / \alpha}\left(\left(\frac{3}{2}\left(1+\frac{4}{2-\alpha} \log \frac{2}{2-\alpha}\right) \log \left(1+\frac{8}{2-\alpha} \log \frac{2}{2-\alpha}\right)\right) \vee \frac{4}{\alpha} \vee\left(6 e^{2}\right)\right)^{1 / \alpha}$.

Proof. Using the notation of the proof of Theorem 5.2 we have

$$
D_{y} g\left(F_{R}\right)(\omega) \leq\left|D_{y} F\left(\omega_{R}\right)\right| \leq c|y|_{X}, \quad P(d \omega) \otimes \nu(d y) \text { a.e. }
$$

where $g(x)=\left(x-m\left(F_{R}\right)\right)^{+} \wedge r$. Thus

$$
\begin{aligned}
& \sup _{y \in B_{X}(0, R)} D_{y} g\left(F_{R}\right) \leq c R, \quad P-\text { a.s. } \\
& \left\|D g\left(F_{R}\right)\right\|_{L^{\infty}\left(\Omega^{X}, L^{2}\left(\nu_{R}\right)\right)}^{2} \leq \frac{c^{2} \sigma\left(S^{n-1}\right)}{2-\alpha} R^{2-\alpha}, \\
& \left\|D g\left(F_{R}\right)\right\|_{L^{\infty}\left(\Omega^{X}, L^{3}\left(\nu_{R}\right)\right)}^{3} \leq \frac{c^{3} \sigma\left(S^{n-1}\right)}{3-\alpha} R^{3-\alpha},
\end{aligned}
$$

and

$$
\left\|D g\left(F_{R}\right)\right\|_{L^{\infty}\left(\Omega^{X}, L^{4}\left(\nu_{R}\right)\right)}^{4} \leq \frac{c^{4} \sigma\left(S^{n-1}\right)}{4-\alpha} R^{4-\alpha} .
$$

We now apply Lemma 5.5 to $\nu_{R}$ and $F_{R}$ with

$$
K=c R, \quad \alpha_{2}=\frac{c^{2} \sigma\left(S^{n-1}\right)}{2-\alpha} R^{2-\alpha}, \quad \alpha_{3}=\frac{c^{3} \sigma\left(S^{n-1}\right)}{3-\alpha} R^{3-\alpha}, \quad \alpha_{4}=\frac{c^{4} \sigma\left(S^{n-1}\right)}{4-\alpha} R^{4-\alpha} .
$$

Using (5.6), equation (5.15) reads

$$
\varphi(s c R):=e^{s c R}-\frac{2 s c R}{2-\alpha}-1=0 .
$$


Since for all $\alpha \in(0,2), \varphi\left(\log \frac{2}{2-\alpha}\right) \leq 0$ and $\varphi\left(2 \log \frac{2}{2-\alpha}\right) \geq 0$ we have

$$
\log \frac{2}{2-\alpha} \leq s_{0} c R \leq 2 \log \frac{2}{2-\alpha}
$$

so that for $x_{0}=3 s_{0}\left(\alpha_{2}-\frac{\alpha_{4}}{K^{2}}\right)=\frac{6 c^{2} \sigma\left(S^{n-1}\right) R^{2-\alpha}}{(2-\alpha)(4-\alpha)} s_{0}$, we have

$$
c \sigma\left(S^{n-1}\right) R^{1-\alpha} \frac{3}{2(2-\alpha)} \log \frac{2}{2-\alpha} \leq x_{0} \leq c \sigma\left(S^{n-1}\right) R^{1-\alpha} \frac{6}{2-\alpha} \log \frac{2}{2-\alpha}
$$

For

$$
r \geq 2 x_{0}=\frac{12 c^{2} \sigma\left(S^{n-1}\right) R^{2-\alpha}}{(2-\alpha)(4-\alpha)} s_{0}
$$

we get from Lemma 5.5 .

$$
\begin{aligned}
& P\left(F_{R}-m\left(F_{R}\right) \geq r\right) \leq P\left(g\left(F_{R}\right)-E\left[g\left(F_{R}\right)\right] \geq r / 2\right) \\
& \quad \leq K_{0} \exp \left(\frac{r}{2 c R}-\left(\frac{r}{2 c R}+3 \frac{\sigma\left(S^{n-1}\right)}{(4-\alpha) R^{\alpha}}\right) \log \left(1+\frac{(4-\alpha) r}{6 \sigma\left(S^{n-1}\right) c R^{1-\alpha}}\right)\right)
\end{aligned}
$$

with from (5.18) and (6.6):

$K_{0}$

$$
\begin{aligned}
& =\exp \left(-\frac{x_{0}}{c R}+\left(\frac{x_{0}}{c R}+3 \frac{\sigma\left(S^{n-1}\right)}{(4-\alpha) R^{\alpha}}\right) \log \left(1+\frac{(4-\alpha) x_{0}}{3 \sigma\left(S^{n-1}\right) c R^{1-\alpha}}\right)-\frac{(2-\alpha)(4-\alpha) x_{0}^{2}}{12 \sigma\left(S^{n-1}\right) c^{2} R^{2-\alpha}}\right) \\
& \leq \exp \left(\frac{3 \sigma\left(S^{n-1}\right)}{2 R^{\alpha}}\left(1+\frac{4}{2-\alpha} \log \frac{2}{2-\alpha}\right) \log \left(1+\frac{8}{2-\alpha} \log \frac{2}{2-\alpha}\right)\right) .
\end{aligned}
$$

Hence under the condition

$$
\sigma\left(S^{n-1}\right) R^{-\alpha} \leq \frac{2}{3\left(1+\frac{4}{2-\alpha} \log \frac{2}{2-\alpha}\right) \log \left(1+\frac{8}{2-\alpha} \log \frac{2}{2-\alpha}\right)}
$$

we get $K_{0} \leq e$ and

$$
\begin{aligned}
2 x_{0} & \leq 12 c \sigma\left(S^{n-1}\right) R^{1-\alpha} \frac{1}{2-\alpha} \log \frac{2}{2-\alpha} \\
& \leq 4 c R \frac{\frac{2}{2-\alpha} \log \frac{2}{2-\alpha}}{\left(1+\frac{4}{2-\alpha} \log \frac{2}{2-\alpha}\right) \log \left(1+\frac{8}{2-\alpha} \log \frac{2}{2-\alpha}\right)} \\
& \leq c R,
\end{aligned}
$$

i.e. $r \geq 2 x_{0}$ with $r=2 c R$. Then from (6.7) and $K_{0} \leq e$ we get

$$
P\left(F_{R}-m\left(F_{R}\right) \geq 2 c R\right) \leq \exp \left(2-\left(1+\frac{3 \sigma\left(S^{n-1}\right)}{(4-\alpha) R^{\alpha}}\right) \log \left(1+\frac{(4-\alpha) R^{\alpha}}{3 \sigma\left(S^{n-1}\right)}\right)\right)
$$




$$
\begin{aligned}
& \leq e^{2}\left(1+\frac{(4-\alpha) R^{\alpha}}{3 \sigma\left(S^{n-1}\right)}\right)^{-1} \\
& \leq \frac{3 e^{2} \sigma\left(S^{n-1}\right)}{(4-\alpha) R^{\alpha}} \\
& \leq \frac{3 e^{2} \sigma\left(S^{n-1}\right)}{2 R^{\alpha}} \\
& =\frac{3}{2} e^{2} \alpha \gamma(R)
\end{aligned}
$$

as long as (6.8) holds. In order to control $P\left(F_{R}-m(F) \geq x\right)$ from (6.9), we need to control $m\left(F_{R}\right)-m(F)$. For this we apply Lemma 5.1 with $\tilde{\beta}(R)=2 c R, \tilde{\gamma}(R)=$ $\frac{3}{2} e^{2} \alpha \gamma(R)$,

$$
R_{0}=\left(\frac{3}{2} \sigma\left(S^{n-1}\right)\left(1+\frac{4}{2-\alpha} \log \frac{2}{2-\alpha}\right) \log \left(1+\frac{8}{2-\alpha} \log \frac{2}{2-\alpha}\right)\right)^{1 / \alpha}
$$

and (6.10). This yields, with $x=4 c R$ :

$$
m\left(F_{R}\right)-m(F) \leq x / 2
$$

and

$$
P\left(F_{R}-m(F) \geq x\right) \leq P\left(F_{R}-m\left(F_{R}\right) \geq x / 2\right) \leq \frac{3}{2} e^{2} \sigma\left(S^{n-1}\right)\left(\frac{4 c}{x}\right)^{\alpha},
$$

provided

$$
R \geq \max \left(R_{0}, \gamma^{-1}(\delta), \gamma^{-1}\left(\frac{2}{3 \alpha e^{2}}\left(\frac{1}{2}-\delta\right)\right)\right),
$$

for any given $\delta \in(0,1 / 2)$. When $x=4 c R$, this estimate together with

$$
P\left(\left\{\omega \in \Omega^{X}: \omega_{R}^{c} \neq \emptyset\right\}\right) \leq \gamma(R)=\frac{\sigma\left(S^{n-1}\right) R^{-\alpha}}{\alpha},
$$

gives, using (5.5):

$$
\begin{aligned}
P(F-m(F) \geq x) & =P\left(F-m(F) \geq x, \omega_{R}^{c}=\emptyset\right)+P\left(F-m(F) \geq x, \omega_{R}^{c} \neq \emptyset\right) \\
& \leq \sigma\left(S^{n-1}\right)\left(\frac{1}{\alpha}+\frac{3}{2} e^{2}\right)\left(\frac{x}{4 c}\right)^{-\alpha}
\end{aligned}
$$

as long as (6.8) and (6.11) hold. Now, conditions (6.8) and (6.11) can be rewritten in terms of $x$ as

$$
x \geq 4 c\left(\frac{3}{2} \sigma\left(S^{n-1}\right)\left(1+\frac{4}{2-\alpha} \log \frac{2}{2-\alpha}\right) \log \left(1+\frac{8}{2-\alpha} \log \frac{2}{2-\alpha}\right)\right)^{1 / \alpha}
$$


and

$$
x \geq 4 c \max \left(\left(\frac{\sigma\left(S^{n-1}\right)}{\alpha \delta}\right)^{1 / \alpha},\left(\frac{3 \sigma\left(S^{n-1}\right) e^{2}}{2(1 / 2-\delta)}\right)^{1 / \alpha}\right),
$$

When e.g. $\delta=1 / 4$, the range of (6.12) can be written

$x \geq 4 c \sigma\left(S^{n-1}\right)^{1 / \alpha}\left(\left(\frac{3}{2}\left(1+\frac{4}{2-\alpha} \log \frac{2}{2-\alpha}\right) \log \left(1+\frac{8}{2-\alpha} \log \frac{2}{2-\alpha}\right)\right) \vee \frac{4}{\alpha} \vee\left(6 e^{2}\right)\right)^{1 / \alpha}$.

Using Lemma [5.6] instead of Lemma[5.5, we can state a similar deviation result under a slight better range condition on $x$, in case $\alpha \in[1,2)$.

Theorem 6.2 Assume that $\alpha \geq 1$ and let $F: \Omega^{X} \rightarrow \mathbb{R}$ such that

$$
\left|D_{y} F(\omega)\right| \leq c|y|_{X}, \quad P(d \omega) \otimes \nu(d y)-\text { a.e. },
$$

with $c>0$. Then we have

$$
P(F-m(F) \geq x) \leq \sigma\left(S^{n-1}\right)\left(1+\frac{e^{2}}{2}\right) \frac{(4 c)^{\alpha}}{x^{\alpha}},
$$

for all

$x \geq 4 c \sigma\left(S^{n-1}\right)^{1 / \alpha}\left(\left(\left(1+\frac{2}{2-\alpha} \log \frac{1}{2-\alpha}\right) \log \left(1+\frac{4}{2-\alpha} \log \frac{1}{2-\alpha}\right)\right) \vee\left(4 e^{2}\right)\right)^{1 / \alpha}$.

Proof. We sketch the modifications of the proof, following the argument of Theorem 6.1 and applying Lemma 5.6 instead of Lemma 5.5 to $\nu_{R}$ and $F_{R}$, with

$$
K=c R, \quad \alpha_{2}=\frac{c^{2} \sigma\left(S^{n-1}\right)}{2-\alpha} R^{2-\alpha}, \quad \alpha_{3}=\frac{c^{3} \sigma\left(S^{n-1}\right)}{3-\alpha} R^{3-\alpha} .
$$

Under the condition

$$
\sigma\left(S^{n-1}\right) R^{-\alpha} \leq \frac{1}{2\left(1+\frac{2}{2-\alpha} \log \frac{1}{2-\alpha}\right) \log \left(1+\frac{4}{2-\alpha} \log \frac{1}{2-\alpha}\right)}
$$

and since

$$
\frac{2 \alpha_{3}}{c^{2} R^{2}} \frac{1}{2-\alpha} \log \frac{1}{2-\alpha} \leq x_{0} \leq \frac{4 \alpha_{3}}{c^{2} R^{2}} \frac{1}{2-\alpha} \log \frac{1}{2-\alpha},
$$


we have $K_{0} \leq e$ and also $x_{0} \leq c R$. Using (5.6) with $r=2 c R \geq 2 x_{0}$, we get applying Lemma 5.6.

$$
\begin{aligned}
P\left(F_{R}-m\left(F_{R}\right) \geq 2 c R\right) & \leq \exp \left(2-\left(1+2 \frac{\alpha_{3}}{c^{3} R^{3}}\right) \log \left(1+\frac{2 c^{3} R^{3}}{\alpha_{3}}\right)\right) \\
& \leq e^{2}\left(1+\frac{2 c^{3} R^{3}}{\alpha_{3}}\right)^{-1} \\
& \leq \frac{e^{2} \alpha_{3}}{2 c^{3} R^{3}} \\
& \leq \frac{e^{2} \sigma\left(S^{n-1}\right)}{2 R^{\alpha}} \\
& =\frac{e^{2} \alpha}{2} \gamma(R),
\end{aligned}
$$

as long as 6.15 holds. Finally, applying Lemma 5.1 with $\tilde{\beta}(R)=2 c R, \tilde{\gamma}(R)=$ $e^{2} \alpha \gamma(R) / 2$ and condition (5.1) given by (6.17), with $x=4 c R$, derive $m\left(F_{R}\right)-m(F) \leq$ $x / 2$, and

$$
P\left(F_{R}-m(F) \geq x\right) \leq P\left(F_{R}-m\left(F_{R}\right) \geq x / 2\right) \leq \frac{e^{2} \sigma\left(S^{n-1}\right)}{2}\left(\frac{4 c}{x}\right)^{\alpha}
$$

provided moreover for any, $0<\delta<1 / 2$,

$$
R \geq \max \left(\gamma^{-1}(\delta), \gamma^{-1}\left(\frac{2}{\alpha e^{2}}\left(\frac{1}{2}-\delta\right)\right)\right) .
$$

With $x=4 c R$, this estimate together with (6.2) gives, using $\alpha \geq 1$ and (5.5)):

$$
\begin{aligned}
P(F-m(F) \geq x) & =P\left(F-m(F) \geq x, \omega_{R}^{c}=\emptyset\right)+P\left(F-m(F) \geq x, \omega_{R}^{c} \neq \emptyset\right) \\
& \leq \sigma\left(S^{n-1}\right)\left(1+\frac{e^{2}}{2}\right)\left(\frac{x}{4 c}\right)^{-\alpha}
\end{aligned}
$$

as long as (6.15) and (6.18) hold. Now, conditions (6.15) and (6.18) can be rewritten in terms of $x$ as (6.14) with e.g. $\delta=1 / 4$.

Finally, we extend a recent result of [16] to Poisson functionals under stable intensity measures.

Theorem 6.3 Let $F: \Omega^{X} \rightarrow \mathbb{R}$ such that for some $c>0$,

$$
\left|D_{y} F(\omega)\right| \leq c|y|_{X}, \quad P(d \omega) \otimes \nu(d y)-\text { a.e. }
$$


1) Let $\varepsilon>0$, then if $\alpha$ is sufficiently close to 2 ,

$$
P(F-m(F) \geq x) \leq(\varepsilon+\sqrt{e}) \exp \left(-\frac{(2-\alpha) x^{\alpha}}{2(4 c)^{\alpha} \sigma\left(S^{n-1}\right)}\right),
$$

provided

$$
\frac{2 \sigma\left(S^{n-1}\right)(4 c)^{\alpha}}{2-\alpha} \log (4(1+\sqrt{e})) \leq x^{\alpha} \leq \frac{\sigma\left(S^{n-1}\right)(4 c)^{\alpha}}{2(2-\alpha)} \frac{\log \left(\frac{1}{2-\alpha}\right)}{3-\alpha} .
$$

2) Let $b>3, \varepsilon>0$, and $x=4 b c \sigma\left(S^{n-1}\right) \frac{1}{2-\alpha} \log \frac{1}{2-\alpha}$. For $\alpha$ close enough to 2 we have

$P(F-m(F) \geq x) \leq \frac{(4 c)^{\alpha} \sigma\left(S^{n-1}\right)}{x^{\alpha}}\left(\frac{1}{\alpha}+(2+\varepsilon) \exp \left(\frac{(2+\varepsilon)(4 c)^{\alpha} \sigma\left(S^{n-1}\right) g(2-\alpha)}{x^{\alpha}}\right)\right)$

where $g(x)=\left(\frac{1}{x} \log \frac{1}{x}\right) \log \left(\frac{1}{x} \log \frac{1}{x}\right)$.

Proof. We follow [16] as in the proofs of Theorem 5.2 and Proposition 6.2 above. First, using the same notation as before, we have:

$$
\begin{aligned}
P\left(F_{R}-m\left(F_{R}\right) \geq r\right) & \leq P\left(g\left(F_{R}\right)-E\left[g\left(F_{R}\right)\right] \geq r / 2\right) \\
& \leq \exp \left(-\int_{0}^{r / 2} h_{R}^{-1}(s) d s\right), \quad 0<x<h_{R}\left(t_{0}^{-}\right),
\end{aligned}
$$

with

$$
\begin{aligned}
h_{R}(s) & \leq\left(\alpha_{2}-\frac{\alpha_{3}}{K}\right) s+\frac{\alpha_{3}}{K^{2}}\left(e^{s K}-1\right) \\
& =\frac{c^{2} \sigma\left(S^{n-1}\right) R^{2-\alpha}}{(2-\alpha)(3-\alpha)} s+\left(e^{s c R}-1\right) \frac{c \sigma\left(S^{n-1}\right) R^{1-\alpha}}{3-\alpha}
\end{aligned}
$$

since again

$$
\alpha_{2}=\frac{c^{2} \sigma\left(S^{n-1}\right)}{2-\alpha} R^{2-\alpha}, \quad \alpha_{3}=\frac{c^{3} \sigma\left(S^{n-1}\right)}{3-\alpha} R^{3-\alpha} \quad K=c R,
$$

where (6.23) above comes as in (5.6) in the proof of Theorem 5.2 and (6.24) comes from the proofs of Lemma [5.6] and Proposition 2.2. Following [16], for $\delta, s, R$ satisfying

$$
\frac{e^{s c R}-1}{c s R} \leq \frac{\delta}{2-\alpha}
$$


we have

$$
h_{R}(s) \leq(1+\delta) \frac{c^{2} \sigma\left(S^{n-1}\right) R^{2-\alpha}}{(2-\alpha)(3-\alpha)} s
$$

and

$$
\int_{0}^{y} h_{R}^{-1}(t) d t \geq \frac{(3-\alpha)(2-\alpha) y^{2}}{2(1+\delta) c^{2} \sigma\left(S^{n-1}\right) R^{2-\alpha}}
$$

for all $y$ such that

$$
\frac{(3-\alpha)(2-\alpha) y}{(1+\delta) c^{2} \sigma\left(S^{n-1}\right) R^{2-\alpha}} \leq s
$$

where $s$ satisfies (6.25). Taking for some $A>0, R^{\alpha}=\frac{A \sigma\left(S^{n-1}\right)}{(2-\alpha)(3-\alpha)}$ and $y=R c$, since $s c R=A /(1+\delta)$, 6.25 can be rewritten as

$$
(1+\delta) \frac{e^{\frac{A}{1+\delta}}-1}{A} \leq \frac{\delta}{2-\alpha}
$$

which is satisfied whenever

$$
(1+\delta) \frac{e^{A}}{A} \leq \frac{\delta}{2-\alpha}
$$

Choosing $\delta=\frac{e^{A}(2-\alpha)}{A-e^{A}(2-\alpha)}$ which is positive for $0<a<A<-\log (2-\alpha)$ when $\alpha$ is close enough to 2 , we derive from (6.26) for $a<A<-\log (2-\alpha)$

$$
\exp \left(-\int_{0}^{c R} h_{R}^{-1}(t) d t\right) \leq e^{-\frac{A}{2}} \exp \left(\frac{e^{A}(2-\alpha)}{2}\right)
$$

But since

$$
\lim _{\alpha \rightarrow 2^{-}} \sup _{a<A<-\log (2-\alpha)} e^{-\frac{A}{2}} \exp \left(\frac{e^{A}(2-\alpha)}{2}\right) e^{\frac{A}{2(3-\alpha)}}=\sqrt{e},
$$

for any $\varepsilon \in(0,1)$ and $\alpha$ close to 2 , from (6.24) with $r=2 c R$,

$$
\begin{aligned}
P\left(F_{R}-m\left(F_{R}\right) \geq 2 c R\right) & \leq \exp \left(-\int_{0}^{c R} h_{R}^{-1}(t) d t\right) \leq\left(\sqrt{e}+\frac{\varepsilon}{2}\right) e^{-\frac{A}{2(3-\alpha)}} \\
& \leq\left(\sqrt{e}+\frac{\varepsilon}{2}\right) \exp \left(-\frac{(2-\alpha)}{2 \sigma\left(S^{n-1}\right)} R^{\alpha}\right)
\end{aligned}
$$

for

$$
\frac{a \sigma\left(S^{n-1}\right)}{(2-\alpha)(3-\alpha)}<R^{\alpha}<\frac{\sigma\left(S^{n-1}\right) \log \frac{1}{2-\alpha}}{2(2-\alpha)(3-\alpha)} .
$$

Next, control $m(F)-m\left(F_{R}\right)$ using Lemma 5.1 with $\tilde{\beta}(R)=2 c R$,

$$
\tilde{\gamma}(R)=(1+\sqrt{e}) \exp \left(-\frac{(2-\alpha)}{2 \sigma\left(S^{n-1}\right)} R^{\alpha}\right)
$$


and condition (5.1) given by (with $\varepsilon \leq 2$ ). This yields

$$
m\left(F_{R}\right)-m(F) \leq 2 c R
$$

provided (15.3), rewritten as

$$
R^{\alpha} \geq \max \left(\frac{\sigma\left(S^{n-1}\right)}{\alpha \delta},-\frac{2 \sigma\left(S^{n-1}\right)}{2-\alpha} \log \frac{1 / 2-\delta}{1+\sqrt{e}}\right),
$$

and (6.29) above still hold. Equations (6.28) and (6.30) yield

$$
\begin{aligned}
\left.P\left(F_{R}-m(F) \geq 4 c R\right)\right) & \leq P\left(F_{R}-m\left(F_{R}\right) \geq 2 c R\right) \\
& \leq\left(\sqrt{e}+\frac{\varepsilon}{2}\right) \exp \left(-\frac{(2-\alpha)}{2 \sigma\left(S^{n-1}\right)} R^{\alpha}\right)
\end{aligned}
$$

provided (6.29) and (6.31) hold. Next, when (6.29) holds, (6.2) gives for $\alpha$ close enough to 2 :

$$
P\left(\omega_{R}^{c} \neq \emptyset\right) \leq \frac{\sigma\left(S^{n-1}\right)}{\alpha R^{\alpha}} \leq \frac{\varepsilon}{2} \exp \left(-\frac{(2-\alpha) R^{\alpha}}{2 \sigma\left(S^{n-1}\right)}\right) .
$$

Finally, (5.5) together with (6.32) and (6.33) yields with $x=4 c R$,

$$
P\left(F-E\left[F_{R}\right] \geq x\right) \leq(\sqrt{e}+\varepsilon) \exp \left(-\frac{(2-\alpha) x^{\alpha}}{2(4 c)^{\alpha} \sigma\left(S^{n-1}\right)}\right)
$$

as long as

$$
a \frac{\sigma\left(S^{n-1}\right)(4 c)^{\alpha}}{(2-\alpha)(3-\alpha)} \leq x^{\alpha} \leq(4 c)^{\alpha} \sigma\left(S^{n-1}\right) \frac{\log (1 /(2-\alpha))}{2(2-\alpha)(3-\alpha)},
$$

and

$$
x^{\alpha} \geq(4 c)^{\alpha} \max \left(\frac{\sigma\left(S^{n-1}\right)}{\alpha \delta},-\frac{2 \sigma\left(S^{n-1}\right)}{2-\alpha} \log \frac{1 / 2-\delta}{1+\sqrt{e}}\right),
$$

for any $0<\delta<1 / 2$. Taking $\delta=1 / 4$, conditions (6.34) and (6.35) can be rewritten as

$$
\frac{2 \sigma\left(S^{n-1}\right)(4 c)^{\alpha}}{2-\alpha} \log (4(1+\sqrt{e})) \leq x^{\alpha} \leq \frac{\sigma\left(S^{n-1}\right)(4 c)^{\alpha}}{2(2-\alpha)} \frac{\log (1 /(2-\alpha))}{3-\alpha},
$$

which yields (6.22).

We now deal with the second part of Theorem [6.3, still following [16]. Take for some $b>0, R^{\alpha}=\frac{b \sigma\left(S^{n-1}\right) \log (1 /(2-\alpha))}{2-\alpha}$ and let $A>0$. For $\alpha$ close to 2 and $s c R \geq \log (1 /(2-\alpha))+\log \log (1 /(2-\alpha))+A$ we have

$$
\frac{e^{s c R}-1}{s c R} \geq \frac{1}{(2-\alpha)\left(e^{-A}+\varepsilon\right)}
$$


hence

$$
h_{R}^{-1}(u) \geq \frac{1}{c R} \log \left(1+\frac{(3-\alpha) u}{\left(e^{-A}+1+\varepsilon\right) c \sigma\left(S^{n-1}\right) R^{1-\alpha}}\right)
$$

whenever

$$
u>u_{1}=\frac{\left(1+e^{-A}+\varepsilon\right) c \sigma\left(S^{n-1}\right) e^{A} \log (1 /(2-\alpha))}{(2-\alpha)(3-\alpha) R^{\alpha-1}} .
$$

For $A>0$ small enough and $b>2$, we have $c R>u_{1}$, and integrating (6.36) over $\left[u_{1}, c R\right]$, we obtain

$\int_{u_{1}}^{c R} h_{R}^{-1}(t) d t \geq\left(\left(1+\frac{1}{\theta c R}\right) \log (1+\theta c R)-1\right)-\left(\left(\frac{u_{1}}{c R}+\frac{1}{\theta c R}\right) \log \left(1+\theta u_{1}\right)-\frac{u_{1}}{c R}\right)$

with $\theta=\frac{(3-\alpha) R^{\alpha-1}}{\left(1+e^{-A}+\varepsilon\right) c \sigma\left(S^{n-1}\right)}$. For $\alpha$ close to 2 and $A, \varepsilon>0$ small enough, using (6.23), (6.24), (6.37), we derive

$$
\begin{aligned}
& P\left(F_{R}-m\left(F_{R}\right) \geq 2 c R\right) \\
& \leq \exp \left(-\int_{0}^{c R} h_{R}^{-1}(t) d t\right) \leq \exp \left(-\int_{u_{1}}^{c R} h_{R}^{-1}(t) d t\right) \\
& \leq \frac{(2+\varepsilon) \sigma\left(S^{n-1}\right)}{R^{\alpha}} \exp \left(\frac{(2+\varepsilon) c \sigma\left(S^{n-1}\right)\left(\frac{1}{2-\alpha} \log (1 /(2-\alpha))\right) \log \left(\frac{1}{2-\alpha} \log (1 /(2-\alpha))\right)}{R^{\alpha}}\right) \\
& \leq \frac{(2+\varepsilon) \sigma\left(S^{n-1}\right)}{R^{\alpha}} \exp \left(\frac{(2+\varepsilon) \sigma\left(S^{n-1}\right) g(2-\alpha)}{R^{\alpha}}\right) .
\end{aligned}
$$

with $g(x)=\left(\frac{1}{x} \log \frac{1}{x}\right) \log \left(\frac{1}{x} \log \frac{1}{x}\right)$ and $\varepsilon$ some (new) positive constant. It is easy now to control $m(F)-m\left(F_{R}\right)$ using once more Lemma 5.1 with $\tilde{\beta}(R)=2 c R, \tilde{\gamma}(x)=$ $\alpha^{-1} \sigma\left(S^{n-1}\right) x^{-\alpha}$, and condition (5.1) given by (6.38). This yields

$$
m\left(F_{R}\right)-m(F) \leq 2 c R
$$

as long as

$$
R^{\alpha} \geq \frac{\sigma\left(S^{n-1}\right)}{\alpha \delta}, \quad \text { and } \quad \frac{3 \sigma\left(S^{n-1}\right)}{R^{\alpha}} \exp \left(\frac{3 \sigma\left(S^{n-1}\right) g(2-\alpha)}{R^{\alpha}}\right) \leq 1 / 2-\delta .
$$

Then with $x=4 c R$, (6.38), (6.39) yield

$$
P\left(F_{R}-m(F) \geq x\right) \leq \frac{(2+\varepsilon) \sigma\left(S^{n-1}\right)}{R^{\alpha}} \exp \left(\frac{(2+\varepsilon) \sigma\left(S^{n-1}\right) g(2-\alpha)}{R^{\alpha}}\right)
$$


as long as (6.40) holds. Together with (5.5) and (6.2), this gives

$$
P(F-m(F) \geq x) \leq \frac{\sigma\left(S^{n-1}\right)}{R^{\alpha}}\left(\frac{1}{\alpha}+(2+\varepsilon) \exp \left(\frac{(2+\varepsilon) \sigma\left(S^{n-1}\right) g(2-\alpha)}{R^{\alpha}}\right)\right)
$$

for $x=4 c R$, that is (6.22) as long as (6.40) holds. This latter condition can be rewritten for $\delta \in(0,1 / 2)$ and $b>3$ :

$$
\frac{1}{2-\alpha} \log \frac{1}{2-\alpha} \geq \max \left(\frac{1}{\alpha \delta b},\left(\frac{3}{b(1 / 2-\delta)}\right)^{\frac{b}{b-3}}\right)
$$

which is obviously true for $\alpha$ close enough to 2 since $\alpha, b$ are bounded below and $\frac{b}{b-3}$ is bounded above.

\section{References}

[1] C. Ané and M. Ledoux. On logarithmic Sobolev inequalities for continuous time random walks on graphs. Probab. Theory Related Fields, 116(4):573-602, 2000.

[2] M. Arcones and E. Giné. On decoupling, series expansions and tail behavior processes. J. Theor. Probab., 6:101-122, 1993.

[3] R. Berthuet. Loi du logarithme itéré pour certaines intégrales stochastiques. Ann. Sci. Univ. Clermont-Ferrand II Math., 19:9-18, 1981. Saint-Flour Probability Summer School (Saint-Flour, 1979/1980).

[4] S. Bobkov, F. Götze, and C. Houdré. On Gaussian and Bernoulli covariance representations. Bernoulli, 7(3):439-451, 2001.

[5] C. Borell. Tail probabilities in Gauss space. In Vector space measures and applications I (Proc. Conf., Univ. Dublin, Dublin, 1977), II, volume 644 of Lect. Notes Math., pages 73-82. Springer, Berlin, 1978.

[6] C. Houdré. Remarks on deviation inequalities for functions of infinitely divisible random vectors. Ann. Probab., 30(3):1223-1237, 2002.

[7] C. Houdré and P. Marchal. On the concentration of measure phenomenon for stable and related random vectors. Preprint, 2002, to appear in Annals of Probability.

[8] C. Houdré and N. Privault. Concentration and deviation inequalities in infinite dimensions via covariance representations. Bernoulli, 8(6):697-720, 2002.

[9] C. Houdré and P. Reynaud-Bouret. Concentration for infinitely divisible vectors with independent components. Preprint, 2003.

[10] N. Ikeda and S. Manabe. Asymptotic formulae for stochastic oscillatory integrals. In Asymptotic problems in probability theory: Wiener functionals and asymptotics (Sanda/Kyoto, 1990), volume 284 of Pitman Res. Notes Math. Ser., pages 136-155. Longman Sci. Tech., Harlow, 1993.

[11] P. Imkeller. On exact tails for limiting distributions of $U$-statistics in the second Gaussian chaos. In Chaos expansions, multiple Wiener-Itô integrals and their applications (Guanajuato, 1992), Probab. Stochastics Ser., pages 179-204. CRC, Boca Raton, FL, 1994. 
[12] Z. Jurek and L. Wu. Large deviations for some Poisson random integrals. In Séminaire de Probabilités, XXXIV, volume 1729 of Lecture Notes in Math., pages 185-197. Springer, Berlin, 2000.

[13] M. Ledoux. A note on large deviations for Wiener chaos. In Séminaire de Probabilités, XXIV, 1988/89, volume 1426 of Lecture Notes in Math., pages 1-14. Springer, Berlin, 1990.

[14] M. Ledoux and M. Talagrand. Probability in Banach spaces. Springer-Verlag, 1991.

[15] P. Lévy. Wiener's random function, and other Laplacian random functions. In Proceedings of the Second Berkeley Symposium on Mathematical Statistics and Probability, 1950, pages 171-187, Berkeley and Los Angeles, 1951. University of California Press.

[16] P. Marchal. A note on measure concentration for stable distributions with index close to 2. Preprint, 2004.

[17] H. Matsumoto and S. Taniguchi. Wiener functionals of second order and their Lévy measures. Electron. J. Probab., 7:no. 14, 30 pp. (electronic), 2002.

[18] E. Mayer-Wolf, D. Nualart, and V. Pérez-Abreu. Large deviations for multiple Wiener-Itô integral processes. In Séminaire de Probabilités, XXVI, volume 1526 of Lecture Notes in Math., pages 11-31. Springer, Berlin, 1992.

[19] H. P. McKean. Wiener's theory of nonlinear noise. In Stochastic differential equations (Proc. SIAM-AMS Sympos. Appl. Math., New York, 1972), pages 191-209. SIAM-AMS Proc., Vol. VI. Amer. Math. Soc., Providence, R.I., 1973.

[20] D. Nualart and J. Vives. Anticipative calculus for the Poisson process based on the Fock space. In Séminaire de Probabilités XXIV, volume 1426 of Lecture Notes in Math., pages 154-165. Springer, Berlin, 1990.

[21] D. Nualart and J. Vives. A duality formula on the Poisson space and some applications. In Seminar on Stochastic Analysis, Random Fields and Applications (Ascona, 1993), pages 205213. Birkhäuser, Basel, 1995.

[22] J. Picard. Formules de dualité sur l'espace de Poisson. Ann. Inst. H. Poincaré Probab. Statist., 32(4):509-548, 1996.

[23] N. Privault. Equivalence of gradients on configuration spaces. Random Operators and Stochastic Equations, 7(3):241-262, 1999.

[24] J. Rosiński. Remarks on strong exponential integrability of vector-valued random series and triangular arrays. Ann. Probab., 23(1):464-473, 1995.

[25] K. Sato. Lévy processes and infinitely divisible distributions, volume 68 of Cambridge Studies in Advanced Mathematics. Cambridge University Press, Cambridge, 1999.

[26] D. Surgailis. On multiple Poisson stochastic integrals and associated Markov semi-groups. Probability and Mathematical Statistics, 3:217-239, 1984.

[27] L. Wu. A new modified logarithmic Sobolev inequality for Poisson point processes and several applications. Probab. Theory Related Fields, 118(3):427-438, 2000.

J.-C. B.: Laboratoire de Mathématiques, Université de La Rochelle, Avenue Michel Crépeau, 17042 la Rochelle, France.

jcbreton@univ-lr.fr 
C.H.: Laboratoire D'Analyse et de Mathématiques Appliquées, CNRS UMR 8050, Université Paris XiI, 94010 Créteil Cedex, France, and School of Mathematics, Georgia Institute of Technology, Atlanta, Ga 30332 USA.

houdre@math.gatech.edu

N.P.: Laboratoire de Mathématiques, Université de la Rochelle, Avenue Michel Crépeau, 17042 la Rochelle, France.

nprivaul@univ-lr.fr 